ARTICLE

\title{
Type-I collagen produced by distinct fibroblast lineages reveals specific function during embryogenesis and Osteogenesis Imperfecta
}

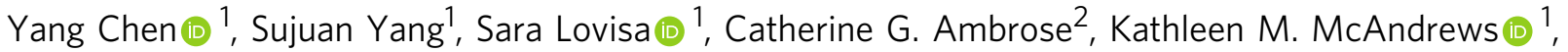 \\ Hikaru Sugimoto ${ }^{1} \&$ Raghu Kalluri (1,3,4凶
}

Type I collagen (Col1) is the most abundant protein in mammals. Col1 contributes to $90 \%$ of the total organic component of bone matrix. However, the precise cellular origin and functional contribution of Col1 in embryogenesis and bone formation remain unknown. Single-cell RNA-sequencing analysis identifies Fap ${ }^{+}$cells and Fsp1 ${ }^{+}$cells as the major contributors of Col1 in the bone. We generate transgenic mouse models to genetically delete Col1 in various cell lineages. Complete, whole-body Col1 deletion leads to failed gastrulation and early embryonic lethality. Specific Col1 deletion in $\mathrm{Fap}^{+}$cells causes severe skeletal defects, with hemorrhage, edema, and prenatal lethality. Specific Col1 deletion in $\mathrm{Fsp}^{+}$cells results in Osteogenesis Imperfecta-like phenotypes in adult mice, with spontaneous fractures and compromised bone healing. This study demonstrates specific contributions of mesenchymal cell lineages to Col1 production in organogenesis, skeletal development, and bone formation/ repair, with potential insights into cell-based therapy for patients with Osteogenesis Imperfecta.

\footnotetext{
${ }^{1}$ Department of Cancer Biology, University of Texas MD Anderson Cancer Center, Houston, TX 77054, USA. ${ }^{2}$ Department of Orthopaedic Surgery, University of Texas Health Science Center at Houston, Houston, TX, USA. ${ }^{3}$ Department of Bioengineering, Rice University, Houston, TX, USA. ${ }^{4}$ Department

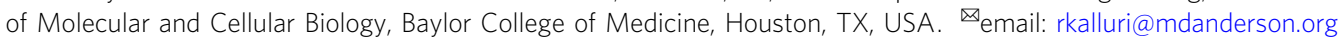


T ype I collagen (Col1), a fibrillar collagen, is the most abundant protein in mammals. The basic functional unit of Coll is a heterotrimer consisting of two $\alpha 1$ chains and one a2 chain that assemble together to form a triple helical structure. Each $\alpha$-chain polypeptide is synthesized in the cytosol and combines with two other $\alpha$-chains to generate a triple-helical type I procollagen (in the format of a1a2a1) with N-terminal and C-terminal propeptides. Subsequently, the procollagen molecule is secreted into the extracellular space where the N-terminal and C-terminal propeptides are cleaved by propeptidases, generating the basic functional heterotrimeric unit of Col1. The Coll triple helical rod-like molecules interact with each other to form fibrils and undergo further crosslinking to form large bundles of fibers.

During embryogenesis, many organs express Coll to likely facilitate cellular migration, differentiation and structural compartmentalization ${ }^{1}$. Insertional mutation of Colla1 gene resulted in complete absence of Coll and led to embryonic lethality ${ }^{2}$. Genetic mutations in Colla1 or Col1a2 genes (or other genes affecting Coll biosynthesis) in humans lead to multiple subtypes of Osteogenesis Imperfecta (OI) syndrome, a bone disease referred to as the "brittle bone disease ${ }^{3-5}$. Previous studies established and investigated various Osteogenesis Imperfecta mouse models using different genetic targeting/manipulating approaches with some success ${ }^{6-19}$. Particularly, recent studies demonstrated that Colla1 heterozygous mutation/deletion in transgenic mouse models lead to Osteogenesis Imperfecta phenotypes $^{14,19}$.

Coll contributes around $90 \%$ of the total organic component of bone matrix. The development, formation, and homeostasis of bone tissue involve mesenchymal cell lineages in the synthesis, deposition, and remodeling of Col1 $1^{4,20}$. These mesenchymal cell lineages include osteoblasts and fibroblasts, which can be differentiated from mesenchymal stem/stromal cells (MSCs) ) $^{3,4,21-25}$. However, the precise contributions of Coll produced by these mesenchymal cell lineages and fibroblasts in the development and function of bones remain unknown. Recent studies, using singlecell RNA sequencing analysis (sc-RNA-seq) and other techniques, highlighted the distinct features and potential functions of various osteogenic cell subpopulations in the bone and bone marrow, shedding some light on the potential mechanisms driving bone homeostasis and repair ${ }^{26-28}$. In this present study, we analyzed sc-RNA-seq dataset on bone and bone marrow stroma of healthy mice $^{27}$, and identified the expression of Colla1 gene dominantly in $\mathrm{Fap}^{+}$(fibroblast activation protein; encoded by Fap) mesenchymal cells, as well as $\mathrm{S100a} 4^{+}$(also known as fibroblastspecific protein 1, Fsp1; encoded by S100a4) mesenchymal cells. Next, we generated novel genetically engineered mouse models (GEMMs) allowing the conditional deletion of Coll specifically in $\mathrm{Fap}^{+}$or $\mathrm{Fspl}^{+}$cells, as well as in other cell lineages. The Colla1 loxP/loxP mouse strain was crossed with multiple Cre recombinase mouse strains including CMV-Cre, Fap-Cre, Fsp1 (S100a4)-Cre, $\alpha S M A$ (Acta2)-Cre, and Cdh5-Cre strains. These mice revealed distinct phenotypes due to Cre recombinase-driven Col1 deletion in targeted cell lineages. Taken together, this study provides comprehensive investigation of the functional roles of Coll produced by various cell lineages in organogenesis, osteogenesis, and bone-related diseases.

\section{Results}

Analysis of single-cell RNA-sequencing of mouse bone and bone marrow identifies type I collagen (Col1)-producing fibroblast/mesenchymal lineages. Bone marrow is one of the sources of mesenchymal lineages, pluripotent cells that differentiate into a variety of cell types such as osteoblasts, chondrocytes, and fibroblasts $3,4,21-25$. To identify the cell populations involved in type I collagen (Col1) production in osteogenesis, we further analyzed the single-cell RNA-sequencing data ${ }^{27}$ of combined cells from mouse bone (including the periosteum) and the bone marrow (Fig. 1a, Supplementary Fig. S1 and S2). Consistent with previous report ${ }^{27}$, six distinct non-hematopoietic cell populations were identified in the bone and the bone marrow (Fig. 1a-c, Supplementary Fig. S1). These are MSCs (expressing Lepr and Cxcl12, also called Cxcl12-abundant-reticular cells) ${ }^{28-30}$, MSC-descendent osteolineage cells (OLCs; expressing Bglap and Runx $2^{31,32}$, chondrocytes (expressing Acan and Col2a1) 33,34 , fibroblasts (expressing S100a4 and Dcn) ${ }^{35}$, bone marrow endothelial cells (BMECs; expressing Cdh5, encoding VE-cadherin) ${ }^{36}$, and smooth-muscle cell/mesenchymal lineage (SMC/mesenchymal) (expressing Acta2 and Myh11) ${ }^{37}$.

Next, we determined that Colla1 is highly expressed by MSCdescendent OLCs and fibroblasts (Fig. 1d). We simultaneously examined the expression of several genes encoding common fibroblast/mesenchymal markers, such as Fap (encoding fibroblast activation protein, Fap), S100a4 (encoding Fibroblastspecific protein 1, Fsp1), and Acta2 (encoding a-smooth muscle actin, aSMA), among the cell populations of bone and bone marrow. Fap expression was observed in cell clusters defined as MSC-descendent OLCs (Fig. 1e), and also observed in chondrocytes, fibroblasts, and MSCs. Fap expression in OLCs was observed in the matrix-producing mature osteoblast subpopulation expressing Bglap (encoding osteocalcin) (Supplementary Fig. 1b), and also the osteoprogenitor cell subpopulation expressing $S p 7$ (Osterix) and Mmp13 (Supplementary Fig. 1c) ${ }^{27,28}$. S100a4 (Fsp1) expression was dominantly detected in all fibroblast clusters ${ }^{27,35}$, but also detected in SMC/mesenchymal cells, chondrocytes, and endothelial cells (Fig. 1f). Acta2 (aSMA) was predominantly expressed in the SMC/mesenchymal cells, and was expressed in other cell types such as fibroblasts, chondrocytes, or osteolineage cells (Fig. 1g). It is important to note that Colla1-expressing cell populations were largely constituted of Fap-expressing cells and Fsp1-expressing cells. Colla1 expression in OLCs was found predominantly in differentiated osteoblasts expressing Bglap (Fig. 1d, Supplementary Fig. 1b), but sparsely in osteoprogenitor cells expressing $S p 7$ and Mmp13 (Supplementary Fig. 1c), which is consistent with recent observations ${ }^{26,27}$. Taken together, these results suggest that Fap-expressing cells and Fsp1expressing cells are likely associated with Coll synthesis in the bones, prompting us to further investigate the functional role of Coll produced by these cell lineages.

Systemic deletion of Colla1 employing CMV-Cre results in failed gastrulation and early embryonic lethality. We generated Colla1 loxp/loxp mice allowing for conditional deletion of floxed Colla1 gene (encoding type I collagen al chain). Complete Coll deletion can be achieved with loss of Colla1 due to the fact that Colla1 chain (encoded by Colla1 gene) is essential for Coll triple helical trimer formation, while Colla2 chain (encoded by Colla 2 gene) alone cannot form Coll trimers. Next, to investigate the functional role of Coll in embryogenesis and organ development, we crossed Collal loxP/loxP mice with CMV-Cre mice (Fig. 2a) to achieve the systemic (whole-body) deletion of Coll (CMV-Cre;Colla1 loxP/loxp, referred to also as Colla $\left.1^{\mathrm{cmvKO}}\right)$. CMV-Cre transgene, under the control of human cytomegalovirus (CMV) minimal promoter, ubiquitously deletes loxP-flanked genes in all tissues including germline cells. Live progeny of Colla1 ${ }^{\mathrm{cmvKO}}$ was not obtained, indicating the embryonic lethality (Fig. 2b). To further determine the stage of lethality, we dissected embryos at E9.5 and E12.5 (Fig. 2c-e). Specifically, Colla ${ }^{\mathrm{cmvKO}}$ embryos at E9.5 revealed a retarded development with the morphology that was normally observed at the E6-E7 gastrulation stage (Fig. 2c). 
a

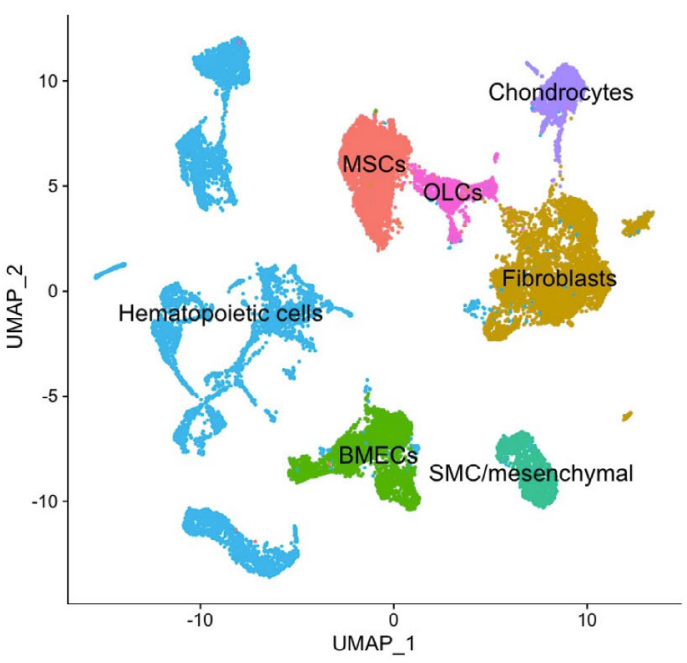

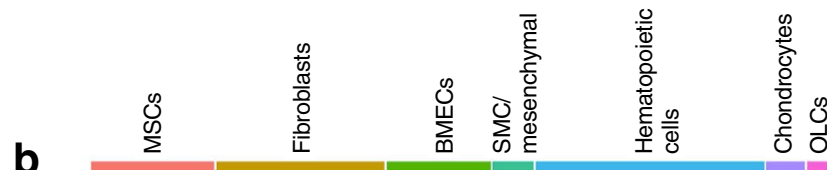

b

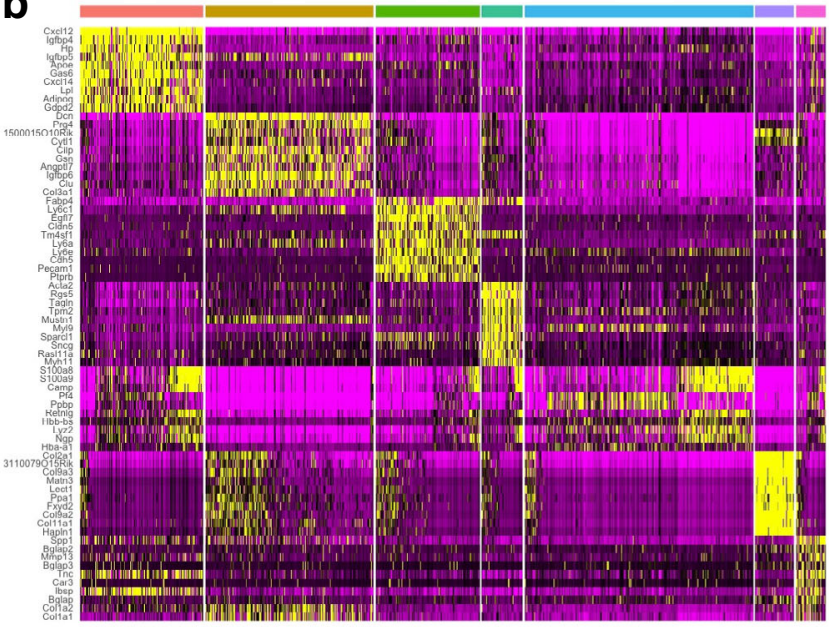

C



Fibroblasts

BMECS
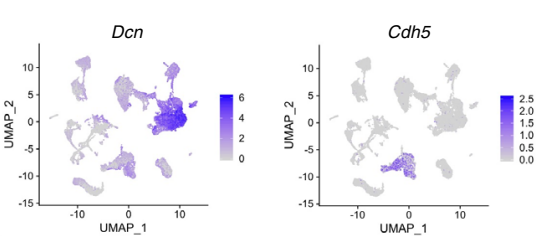

SMC/mesenchymal cells

Chondrocytes

OLCs
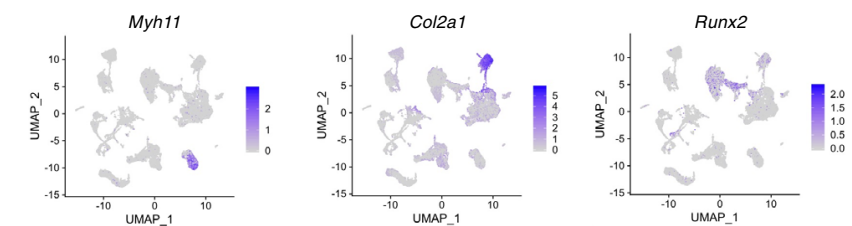

d

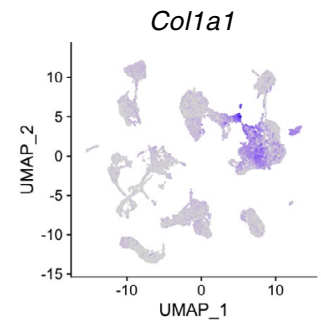

f

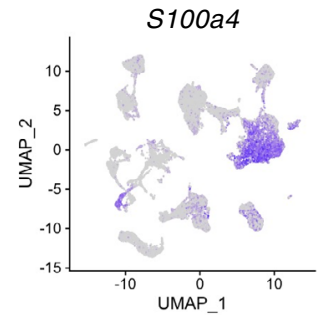

Col1a1
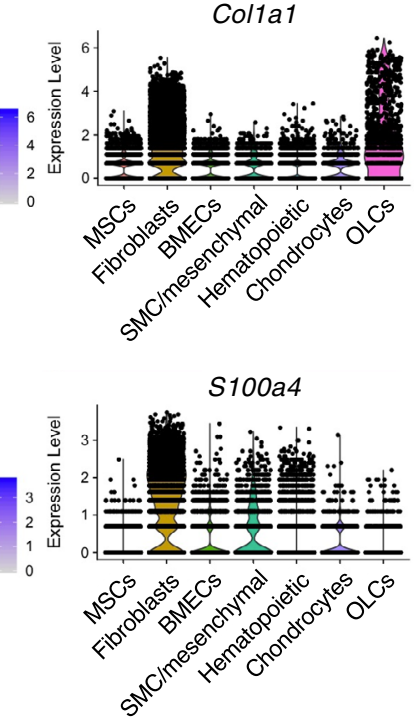

e
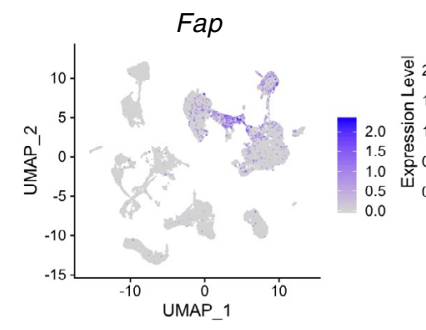

Fap

g

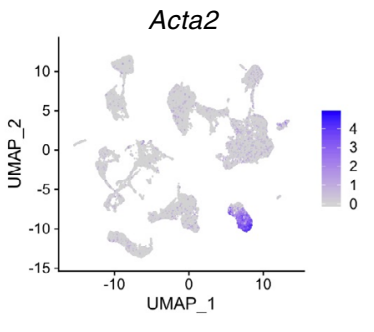

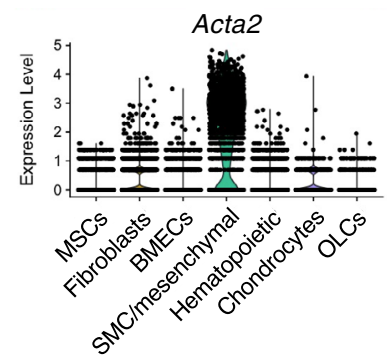

Fig. 1 Single-cell RNA-sequencing analysis identifies Col1-producing cells/lineages in bone and bone marrow fractions. a-c Single-cell RNA-sequencing (sc-RNA-seq) analysis of cell mixture of mouse bone and bone marrow fractions ( $n=6$ mice) from a recently published dataset (GSE128423). Functional clusters of cells were defined with group definition listed, as implemented in the Seurat R package (a). MSCs mesenchymal stem/stromal cells, OLCs osteolineage cells derived from MSCs, BMECs bone marrow endothelial cells, SMC/mesenchymal smooth-muscle cell/mesenchymal lineage. b Heat map showing the scaled expression values of discriminating signature genes for the functional clusters of cells defined above. (c) Expression profile of representative signature gene for each of the non-hematopoietic cell clusters. Continued in Supplementary Fig. 1 and 2. d-g Expression profile of type I collagen $\alpha 1$ chain (Col1a1; d), fibroblast activation protein (Fap; e), fibroblast-specific protein 1 (S100a4; $\mathbf{f}$ ), and $\alpha$-smooth muscle actin (Acta2; $\mathbf{g}$ ) among defined cell clusters shown in UMAP plot and violin plot. 
a

Col1a1cmvko

(CMV-Cre;Col1a 10xP/loxP)

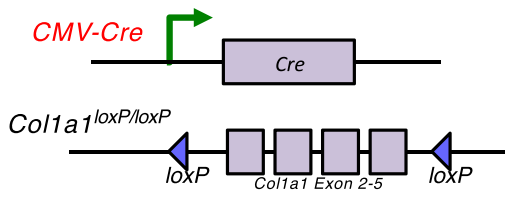

C

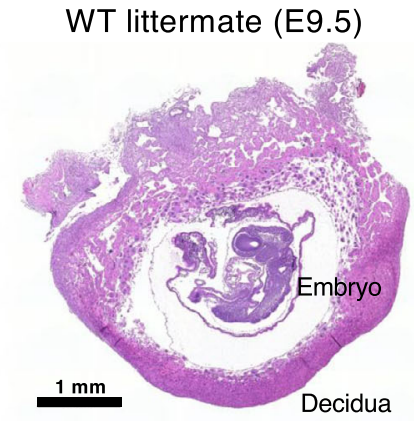

Col1 a1 ${ }^{\text {cmvko }}$ (E9.5)

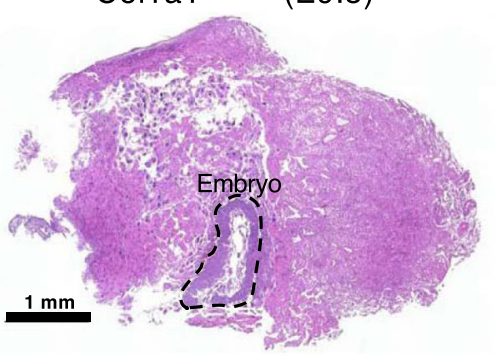

d

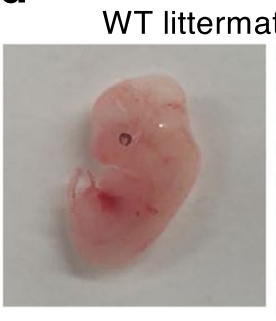

$(E 12.5)$

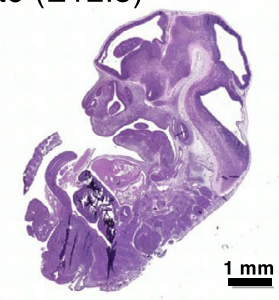

b Genotype distribution of offspring from CMV-Cre;Col1a 110xP/+ $\times$ Col1a110xPlloxp crosses

\begin{tabular}{lcc}
\hline Genotype & Number at weaning (\%) & Expected number (\%) \\
\hline CMV-Cre;Col1a1loxPloxP & $0(0 \%)$ & $19.5(25 \%)$ \\
CMV-Cre;Col1a1 10xP/+ & $18(23.1 \%)$ & $19.5(25 \%)$ \\
Col1a110xPhoxP & $31(39.7 \%)$ & $19.5(25 \%)$ \\
Col1a1 10xP/+ & $29(37.2 \%)$ & $19.5(25 \%)$
\end{tabular}

The expected number was according to the total number of mice and based on the expected Mendelian 1:1:1:1 ratio
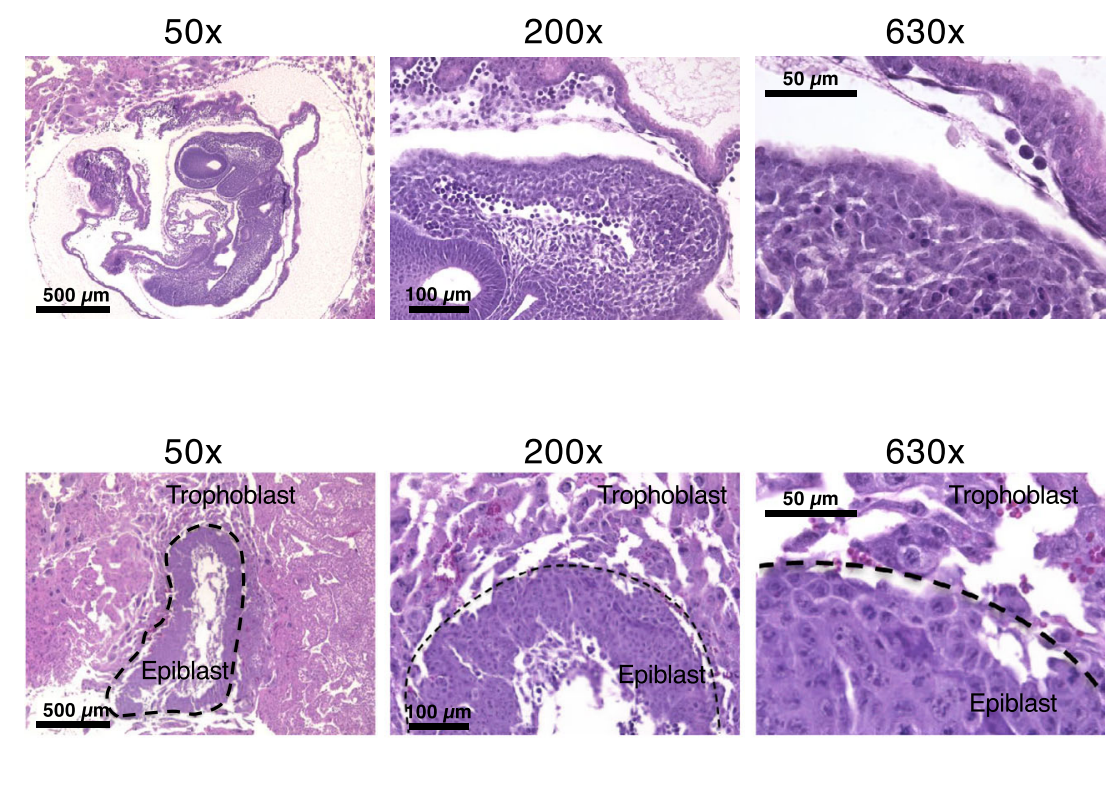
Genotype distribution of offspring from CMV-Cre;Col1a1loxP/+ $\times$ Col1a1loxPlloxP crosses

e
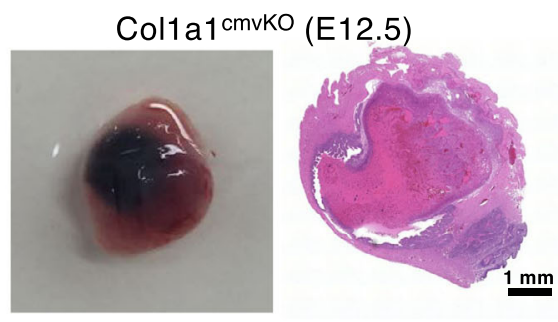

\begin{tabular}{lrc}
\hline Genotype & E9.5 & E12.5 \\
\hline CMV-Cre;Col1a1loxPhoxP & 7 & 3 \\
CMV-Cre;Col1a1loxP/t & 10 & 12 \\
Col1a1loxPhoxP & 9 & 15 \\
Col1a1loxP/t & 12 & 14 \\
\hline DNA for gonotyping
\end{tabular}

DNA for genotyping PCR was isolated from yolk sac of embryos

Fig. 2 Systemic deletion of Col1 by CMV-Cre transgene results in early embryonic lethality. a Genetic strategy to achieve systemic (whole-body) knockout of type I collagen $\alpha 1$ chain (Col1a1) by crossing the Col1a 1oxP/loxP mice with generic CMV-Cre mice. b Genotype distribution in live offspring examined at the time of weaning from the crosses between CMV-Cre;CollalloxP/+ and Colla loxP/loxP mice. c, d Hematoxylin and eosin (H\&E) staining of the embryos of CMV-Cre-negative;Col1alloxP/loxP (WT; wild-type) and CMV-Cre;Col1a1loxP/loxP (Colla1cmvKO) at embryonic day E9.5 (c) and E12.5 (d).

Col1a1 ${ }^{\mathrm{CmvKO}}$ embryo of E9.5 was indicated by dashed line, with labeled epiblast and trophoblast areas. Representative images were shown for WT embryos

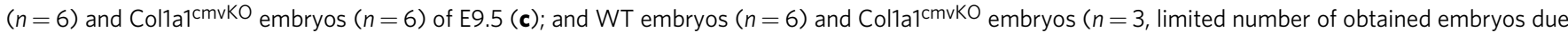
to lethality) of E12.5 (d). e Genotype distribution in live offspring examined at E9.5 and E12.5 from the crosses between CMV-Cre;Col1a loxP/+ and Colla 7loxP/ loxP mice. Scale bars of whole-mount sections, $1 \mathrm{~mm}$; Scale bars at $50 \times$ magnification, $500 \mu \mathrm{m}$; Scale bars at $200 \times$ magnification, $100 \mu \mathrm{m}$; Scale bars at $630 \times$ magnification, $50 \mu \mathrm{m}$.

Our results indicate the essential role of Col1 during gastrulation, consistent with previous observations identifying Coll protein between embryonic endoderm and mesoderm ${ }^{2}$. Moreover, our observations that the whole-body KO of Col1 (truncated Colla1 exons $2-5$ by CMV-Cre) resulted in failed gastrulation, arrested embryonic development at E6-E7, and the death of embryos (Fig. 2d, e), validated previous observations with a mouse model harboring the loss of Col1 by insertional mutation of Col1a1 gene, resulting in embryonic lethality at E12-E14 due to blood vessel rupture $^{2}$. CMV-Cre;Colla1 $1^{\text {loxP/+ }}$ mice harboring heterozygous Colla1 loss revealed minimal abnormalities in their general health, overall survival, or their breeding ability until 6-month age (Supplementary Fig. 3a). Mild spontaneous bone fractures could be observed by X-ray examination in 6-month-old CMV-Cre;Colla1 $1^{\text {loxP/+ }}$ mice (Supplementary Fig. 3b), consistent with previous studies using Osteogenesis Imperfecta type I mouse models 

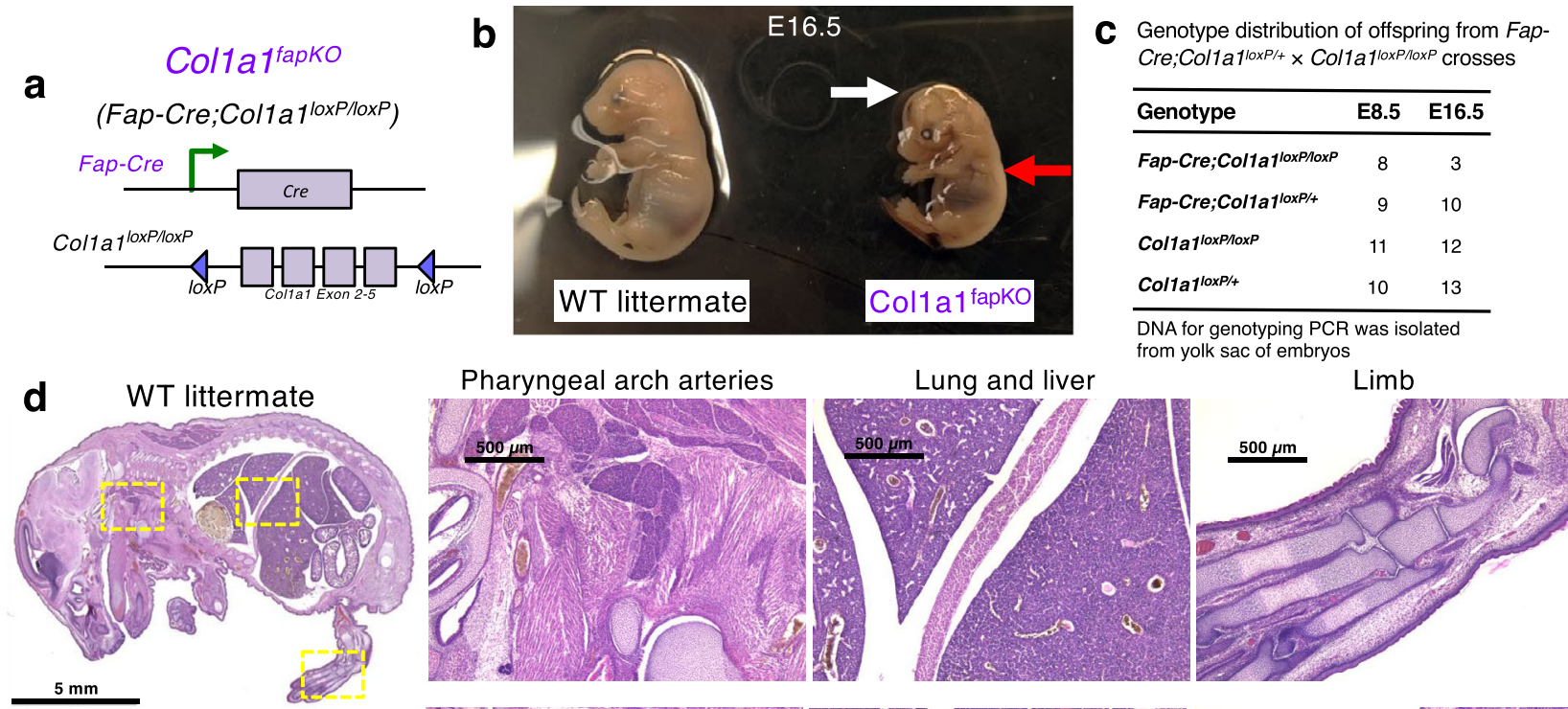

Pharyngeal arch arteries
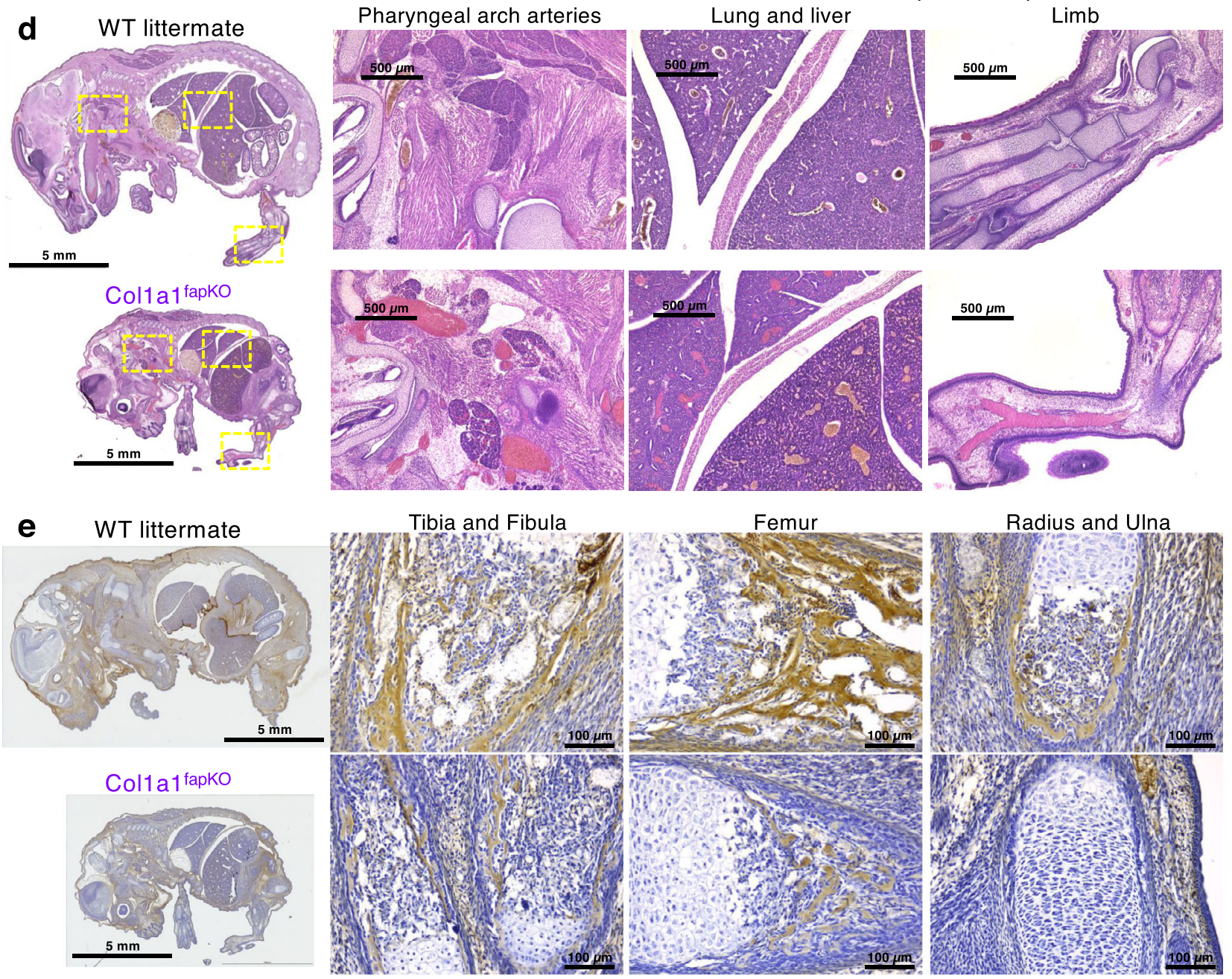

f

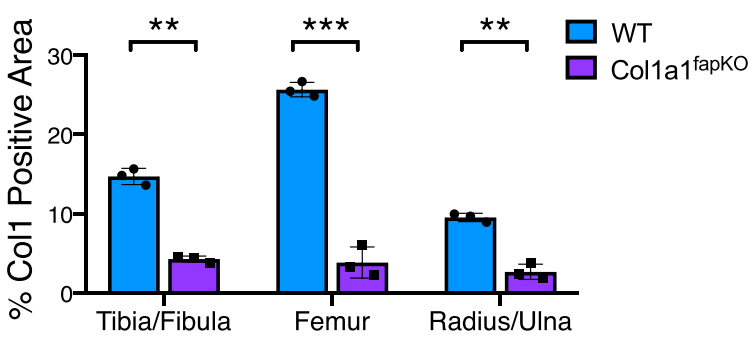

harboring Colla1 heterozygous truncation/mutation ${ }^{14,19}$. Further investigations will be required to evaluate the mechanism by which such Col1a1 haploinsufficiency in CMV-Cre;Col1a1 ${ }^{l o x P} /+$ mice may lead to the phenotype similar to human type I Osteogenesis Imperfecta (OI). g

Genotype distribution of offspring from Fap-Cre;Col1a 1loxP/+ $^{\mathrm{x}}$ Col1a 1loxploxp crosses

\begin{tabular}{lcc}
\hline Genotype & Number at weaning (\%) & Expected number (\%) \\
\hline Fap-Cre;Col1a1loxPloxP & $1(1.3 \%)$ & $19(25 \%)$ \\
Fap-Cre;Col1a1loxP/t & $22(28.9 \%)$ & $19(25 \%)$ \\
Col1a1loxPhoxP & $25(32.9 \%)$ & $19(25 \%)$ \\
Col1a1loxP/+ & $28(36.8 \%)$ & $19(25 \%)$ \\
\hline
\end{tabular}

The expected number was according to the total number of mice and based on the expected Mendelian 1:1:1:1 ratio

Specific deletion of Col1 in $\mathrm{Fap}^{+}$cells leads to impaired skeletal development and embryonic lethality. To test the functional role of Coll produced by the Fap ${ }^{+}$cells in the bone, we generated the Fap-Cre;Collal loxP/loxP (Colla1 fapKO) mice (Fig. 3a). Most Colla1 ${ }^{\text {fapKO }}$ embryos were non-viable past E16.5 
Fig. 3 Specific deletion of Col1 in Fap-lineage cells leads to impaired skeletal development and late embryonic lethality. a Genetic strategy to delete Colla1 specifically in Fap-lineage cells by crossing the Colla7loxP/loxP mice with Fap-Cre mice. b Wild-type (WT; with genotype of Fap-Cre-negative;CollaloxP/loxP) and Colla1fapKO (Fap-Cre;CollalloxP/loxP) embryos at E16.5. The Colla1fapKO embryo exhibits hydrops fetalis (white arrow) and hemorrhage (red arrow). c Genotype distribution in live offspring documented examined at E9.5 and E12.5 from the crosses between Fap-Cre;Colla loxP/+ and Colla 7 loxP/loxP mice. d-f H\&E staining (d) and Col1 immunohistochemistry staining (e) of the embryos of WT and Col1a1 ${ }^{\text {fapKO }}$ at E16.5. Quantification of \% positive area for Col1 staining was based on three mice per group (f). The unpaired, two-tailed $t$ test was used to compare the mean of two independent groups. ${ }^{\star \star} P=0.00116$ (tibia/fibula), ${ }^{\star \star \star} P=0.00057$ (femur), ${ }^{\star \star} P=0.00149$ (radius/ulna). Data are represented as mean \pm SEM. Additional Picrosirius Red staining was shown in Supplementary Fig. 4. g Genotype distribution in live offspring documented at the time of weaning from the crosses between Fap-Cre;Colla $7^{10 x P /+}$ and Colla $7^{10 x P / / 0 x P}$ mice. Scale bars of whole-mount sections, $5 \mathrm{~mm}$; Scale bars at 50x magnification, $500 \mu \mathrm{m}$; Scale bars at 200x magnification, $100 \mu \mathrm{m}$.

and revealed prominent hemorrhage and edema (Fig. 3b), while the number of Colla1 fapKO embryos at E8.5 was comparable to the expected number based on Mendelian ratio (Fig. 3c). Histological analysis further confirmed the hemorrhagic phenotype in major organs and pharyngeal arch arteries in Collal fapKO embryos (Fig. 3d). Significantly decreased collagen deposition in skeletal system was also observed in Colla1 fapKO embryos, as examined by Coll immunohistochemical staining (Fig. 3e, f) as well as Picrosirius Red staining (Supplementary Fig. 4 and 5a). Despite the decreased Coll deposition, the skeletal morphology of Colla1 fapKO embryos did not reveal prominent deformity as compared with WT embryos. Live progeny of Colla1 fapKO genotype was almost never observed with only one exception, indicating that Colla1 ${ }^{\text {fapKO }}$ leads to embryonic lethality with almost $100 \%$ penetrance (Fig. 3g).

Despite the fact that most CollalfapKO embryos died prenatally, we indeed observed one live progeny with Colla $1^{\text {fapKO }}$ genotype. This mouse was drastically smaller in size compared to its littermates with severely defective/fractured skeleton throughout the body (Fig. 4a). Histological analysis further revealed the dramatic fractures in Colla 1 fapKO mouse (Fig. 4b), associated with significantly decreased Coll deposition in the bones (Fig. 4c, d). The fractures in Colla1 $1^{\text {fapKO }}$ mouse was accompanied by the significant accumulation of chondrocytes and cartilage deposition, as examined by $\mathrm{H} \& \mathrm{E}$ and Safranin-O/Fast Green staining (Fig. 4c, d). Prominent deformities were also observed in both the limb skeleton and the axial skeleton of the Collal fapKO mouse (Supplementary Fig. 5b). A lineage-tracing Fap-Cre;LSL-tdTomato mouse model exhibited colocalization between Fap-CretdTomato $^{+}$cells and osteoblast marker osteocalcin (Fig. 4e), consistent with previous sc-RNA-seq analysis showing Fapexpression in osteolineage cells (Fig. 1e). These results do not rule out the possibility that Fap $^{+}$fibroblasts may derive from osteolineage cells or osteoblasts. Taken together, these results demonstrate the fundamental role of $\mathrm{Fap}^{+}$cells in the de novo production of Collin vascular organogenesis and bone development during embryogenesis.

Specific deletion of Col1 in Fsp1 ${ }^{+}$cells leads to Osteogenesis Imperfecta phenotype in mice. To test the function of Coll produced by the $\mathrm{Fsp1}^{+}$cells in the bone stroma, we generated the Fsp1-Cre;Collal loxP/loxP (Colla1 ${ }^{\text {fspKO}}$ ) mice (Fig. 5a). These mice were born normal but Col1 deletion in Fsp1-lineage cells led to the occurrence of spontaneous Osteogenesis Imperfecta (OI) symptoms in adult Colla1 ${ }^{\text {fspKO }}$ mice, which was analogous to the previous observations employing systemic transgenic mice harboring dominant mutations of Col1a1 and Colla2 $2^{6,12,14,15,38,39}$. Colla $1^{\text {fspKO }}$ mice developed OI phenotype with full penetrance, resulting in death of mice at around 6-12 months of age (Fig. 5b). At the age of 6 month, all Collal fspKO mice exhibited the inevitable occurrence of fractures and deformations, frequently observed in the joints, pelvis, ischium, and head of femur (Fig. 5c, d). Such OI-like phenotype of Collal fspKO mice was very similar to the well-characterized Colla2 ${ }^{O I M} / O I M$ mice $^{6}$ and phenotypically resembled the moderate to severe OI type III in humans. As shown in Supplementary Fig. 6a, b as a reference, the Colla2 OIM/OIM mouse model harbors homozygous whole-body point-mutated Colla2 alleles and develops severe OI feature in the bones, similar to the Colla1 ${ }^{\text {fspKO }}$ mice.

Further radiographic analysis revealed that mild deformation of pelvis and ischium was observed as early as 2-3 months of age, and more severe fracture in hindlimbs associated with dysfunction by the 5-8 months or age (Fig. 5d, e). Noticeable deformation of elbow joints can also be seen as early as 3-4 months of age, resulting in forelimb dysfunction by 6-9 months of age (Fig. 5d, e). In contrast, the age-matched wild-type (Fsp1-Cre-negative;Col1a1 loxP/loxP) littermates did not reveal any of the above symptoms (Supplementary Fig. 7a). Of note, Colla1 fspKO mice were born at the expected Mendelian ratio

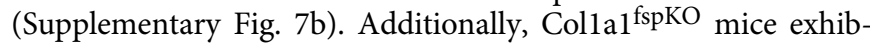
ited no gross physical or behavioral abnormalities before onemonth of age (Fig. 5e, f). Significant body weight loss and hampered movement were observed in all Colla1 ${ }^{\mathrm{fspO}}$ mice by 3-6 months of age (Fig. 5f and Supplementary Movie 1). Female and male Colla1 ${ }^{\mathrm{fspKO}}$ mice revealed similar body weight loss and overall survival (Fig. 5f and Supplementary Fig. 7c). Micro-CT measurements confirmed the significantly decreased bone mineral density and bone material properties in six-month-old Colla $1^{\text {fspKO }}$ mice as compared with age-matched WT littermates (Fig. $5 \mathrm{~g}$ and Supplementary Fig. 7d). Spontaneous fractures in the head of femur were ubiquitously observed in Collal ${ }^{\text {fspKO }}$ mice (Fig. 5g). Biomechanical assays validated the brittleness of femurs from six-month-old Colla $1^{\mathrm{fspKO}}$ mice as compared with WT littermates (Fig. 5h).

Next, we performed histological analysis of joint lesions from Colla1 ${ }^{\text {fspKO }}$ mice (Fig. $6 \mathrm{a}$ and Supplementary Fig. 7e). In the joint lesions with fractures and deformations, we observed significant callus formation composed of chondrocytes and cartilage components adjacent to the fractured areas, as observed by Safranin-O staining of cartilage matrix (Fig. 6a, b). The joint lesions from Collal fspKO mice also revealed significantly decreased overall levels of fibrillar Coll, as examined using circularly polarized light microscopy on Picrosirius Red-stained sections (Fig. 6c). Lineage-tracing Fsp1-Cre;LSL-YFP mouse model revealed Fsp1-Cre-induced YFP expression in the periosteum, connective tissue, and bone marrow (Fig. 6d). Fsp1-Creinduced YFP expression was also observed in osteocalcinexpressing osteoblasts in a mosaic pattern (Fig. 6e). These observations indicate that the phenotypes of Colla1 ${ }^{\text {fspKO }}$ mice may result from the combined effect of Coll deletion in Fsp1 cell lineage including both osteoblasts and other periosteal cells. Collectively, these results establish Colla1 ${ }^{\text {fspKO }}$ mice as a novel cell-specific transgenic mouse model for OI. Our observations that the Colla1 fspKO mice reveal insignificant abnormalities in the bones before 1 month of age and develop OI symptoms after 2-3 months of age, emphasize the crucial role of Col1 produced specifically by Fsp1-lineage cells in regulating proper bone development and healing of fractures. 
a
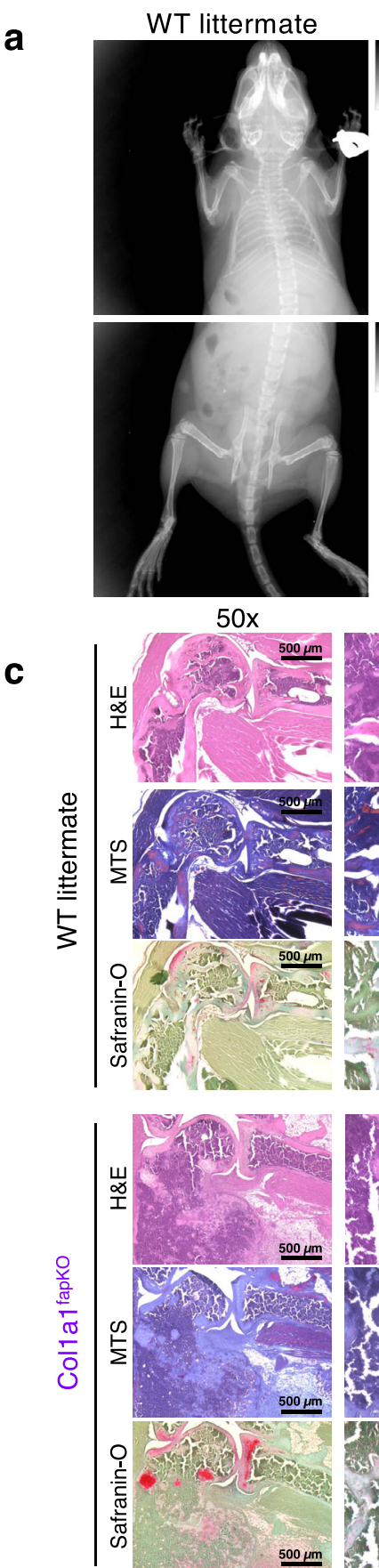

Col1 a1 fapko
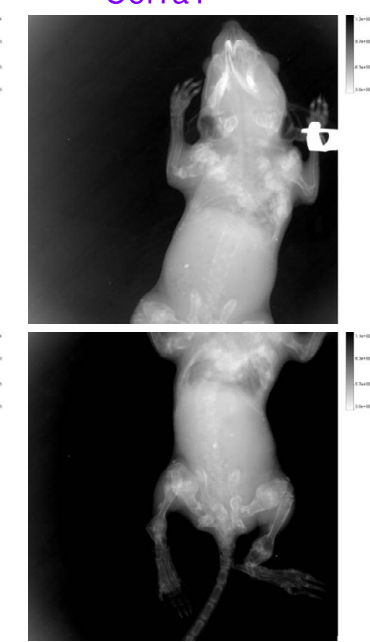

200x
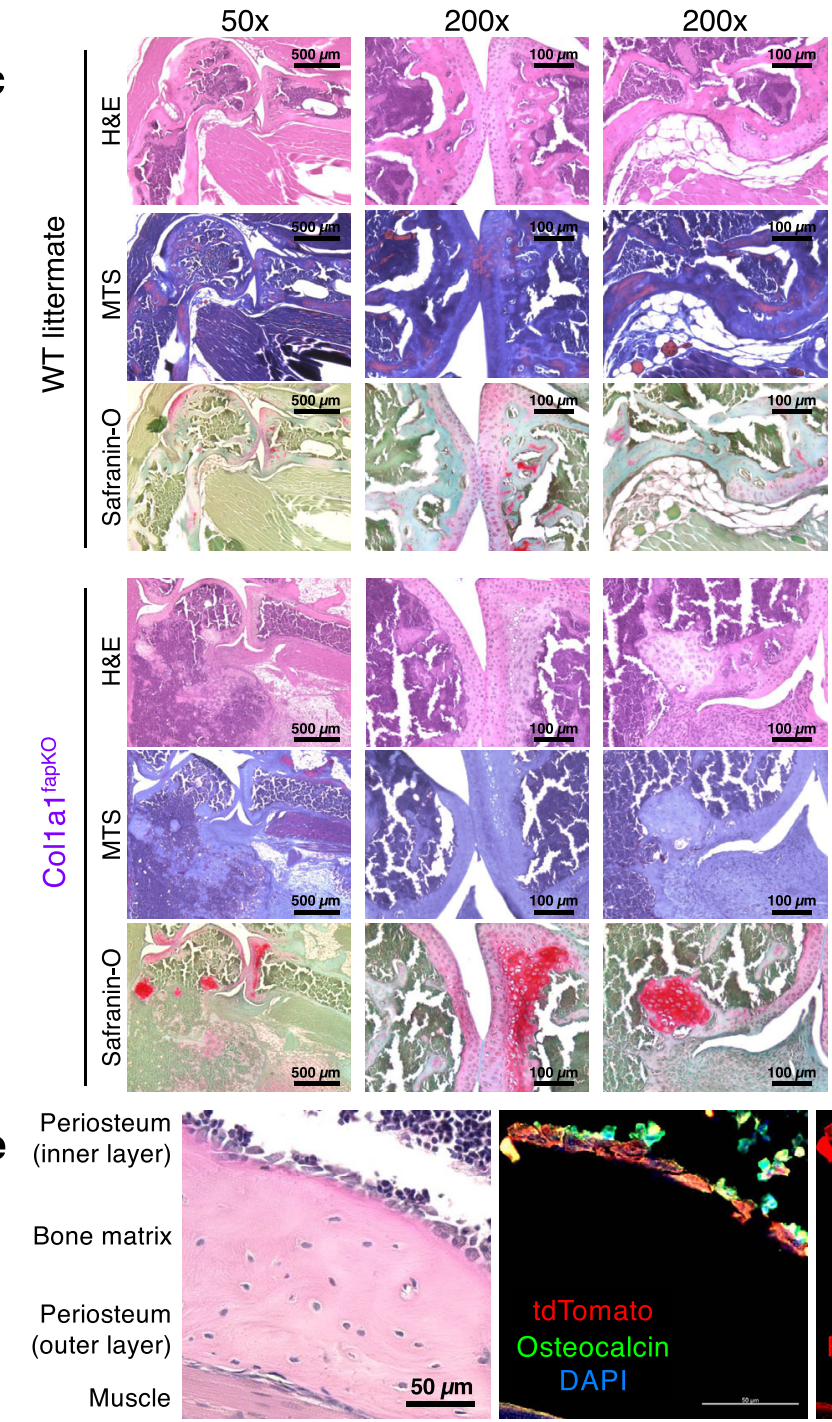

b

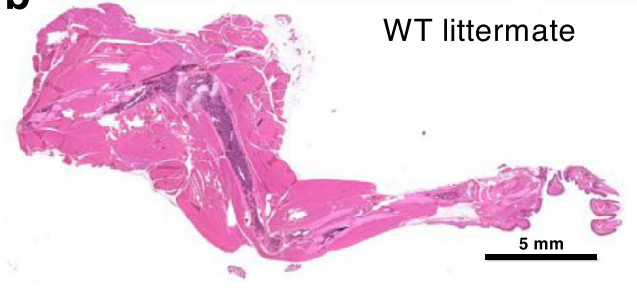

Col1a1 fapko

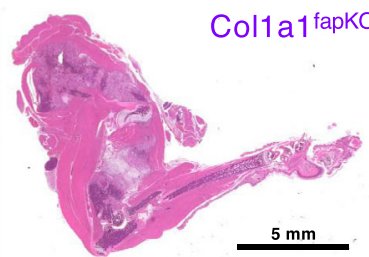

200x
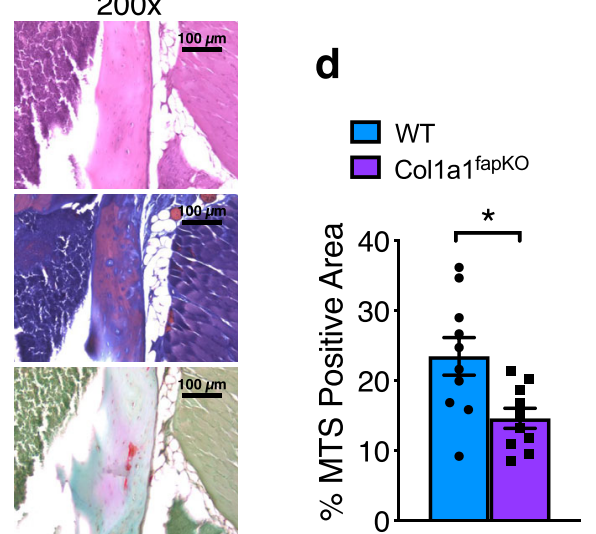

Col1 deletion in Fsp1 ${ }^{+}$cells alters the composition of neutrophils and macrophages in the bone marrow. In order to further investigate the impact of Coll deletion in the $\mathrm{Fsp} 1^{+}$cells on the bone marrow microenvironment, we conducted single-cell RNA-sequencing analysis (sc-RNA-seq) on unfractionated live cell mixture from Colla fspKO $^{\mathrm{sp}}$ mouse bone marrow (Fig. 7a, Supplementary Fig. 8a-c). Colla1 ${ }^{\text {fspKO }}$ mouse bone marrow was

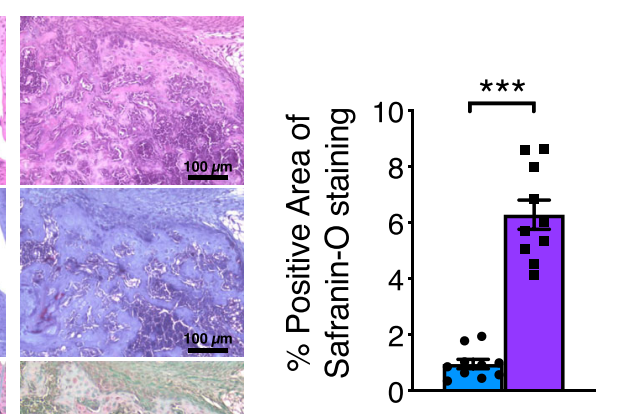

ojm
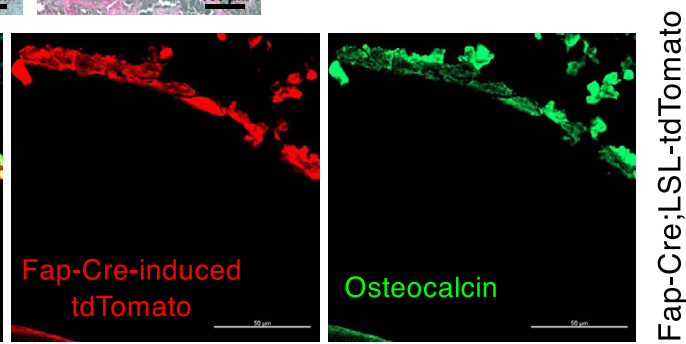

examined at 2-month age (when OI phenotype initiates in these mice), as compared with age-matched wild-type littermate bone marrow. Most of the major cell lineages (such as T cell, B cell, erythroid cell, eosinophil/basophil, and dendritic cell populations) remained largely unchanged in Colla $1^{\mathrm{fspKO}}$ mouse bone marrow (Fig. 7a, b). Interestingly, Colla $1^{\mathrm{fspKO}}$ mouse bone marrow revealed significantly increased percentage of a specific neutrophil 
Fig. 4 The only live offspring of Col1a1fapKO genotype exhibits severe skeletal defects. a Radiograph of three-week-old wild-type (WT, Fap-Crenegative;Col1a1 ${ }^{\text {loxP/loxP }}$ ) and Col1a1 fapKO mice. $\mathbf{b}$ H\&E staining of the forelimb whole-mount sections of 3-week-old WT $(n=3)$ and Col1a1 fapKO $(n=1$, limited by the fact that only one live Col1a1fapKO mouse was ever observed) mice. c H\&E staining, Masson's Trichrome staining (MTS), and Safranin-O/Fast Green staining on serial sections of the forelimbs from three-week-old WT $(n=3)$ and Col1a1 fapKO $(n=1$, limited by the fact that only one live Col1a1 fapKO mouse was ever observed) mice. d Quantification of \% positive area for MTS staining (blue indicating collagen deposition) and Safranin-O/Fast Green staining (red indicating cartilage) based on the staining shown in (c). Quantification of \% positive area for staining was based on 10 bone samples from WT group ( $n=3$ mice) and Col1a 1 fapKO group ( $n=1$ mouse, limited by the fact that only one live Colla fapKO mouse was ever observed). The unpaired, twotailed $t$ test was used to compare the mean of two independent groups. ${ }^{\star} P=0.0116,{ }^{\star \star \star} P=1.11 \mathrm{E}-06$. Data are represented as mean $\pm \mathrm{SEM}$. $\mathbf{e}$ Representative H\&E and immunofluorescence images of humerus serial sections from Fap-Cre;LSL-tdTomato mice ( $n=3$ mice at 2-month-old) stained for osteoblast marker osteocalcin (green) and Fap-Cre-induced tdTomato (red). Scale bars, $50 \mu \mathrm{m}$. Scale bars of whole-mount sections, $5 \mathrm{~mm}$; Scale bars at $50 \times$ magnification, $500 \mu \mathrm{m}$; Scale bars at $200 \times$ magnification, $100 \mu \mathrm{m}$.

cluster ("Neutrophil-1" cluster, Fig. 7a, b) when compared to wild-type littermate bone marrow. This uniquely increased neutrophil cluster revealed high expression of matrix metalloproteinase 9 (MMP9) (Fig. 7c, d), which has been previously shown to regulate bone fracture repair $^{26,40,41}$. This MMP9-high Neutrophil-1 subpopulation highly expressed $M m p 8, C x c r 2$, and Retnlg (Fig. 7c, d, and Supplementary Fig. 8d, e), as well as the general neutrophil markers such as S100a8, Ly6g, and Itgam/ CD11b (Supplementary Fig. 8d). The top upregulated genes in the Neutrophil-1 cluster of Colla $1^{\text {fspKO }}$ bone marrow (in comparison with WT bone marrow) were largely anti-inflammatory neutrophil marker genes including $L y 6 g, L y 6 c 1, L y 6 c 2$, and Cxcr2 (Fig. 7e, f). The significant enrichment of $\mathrm{Ly} 6 \mathrm{G}^{+}$neutrophils in Collal fspKO bone marrow was also confirmed by immunofluorescence staining (Fig. 7g). Ingenuity Pathway Analysis (IPA) of signature genes in the Neutrophil-1 cluster (Supplementary Fig. 8e) revealed the correlation with neutrophil degranulation/ activation. Additionally, the monocyte/macrophage cluster of Colla1 ${ }^{\text {fspKO }}$ mouse bone marrow also revealed a noticeable shift towards Adgre1 (F4/80)-expressing subpopulation (Supplementary Fig. 8f), while the overall percentage of monocyte/macrophage cluster exhibiting a mild increase.

In conclusion, these results suggest an involvement of neutrophils and monocytes/macrophages in response to the bone fractures during early OI pathogenesis in Colla1 $1^{\text {fspKO }}$ mice ${ }^{42-46}$.

Normal phenotype of mice with specific deletion of Col1 in $\mathrm{aSMA}^{+}$mesenchymal cells or $\mathrm{Cdh}^{+}$endothelial cells. Additionally, we also generated the $\alpha S M A-C r e$;Colla1 loxP/loxP (Colla1 ${ }^{\text {smaKO}}$ ) and Cdh5-Cre;Collal loxP/loxP (Colla1 ${ }^{\text {cdh5KO})}$ mouse models, with the potential to delete Coll in aSMA ${ }^{+}$ mesenchymal cells and Cdh5(VE-Cadherin) ${ }^{+}$endothelial cells, respectively. Both Colla1 ${ }^{\text {smaKO }}$ and Colla1 $1^{\text {cdh } 5 \mathrm{KO}}$ mice were born at the expected Mendelian ratio, when compared to their littermate controls (Supplementary Fig. 9a, b). These mice exhibit normal tissue throughout the body, normal litter size, and unaltered life span, without any overt phenotype. Fractures were not observed in either Colla1 $1^{\text {smaKO }}$ or Colla1 $1^{\text {cdh5KO }}$ mice at 12month age (Supplementary Fig. 9c, d). The body weight of Colla1 $1^{\text {smaKO }}$ or Colla1 $1^{\text {cdh5KO }}$ mice at 12 -month age was identical to that of their wild-type littermates (Supplementary Fig. 9e, f). These results were not surprising, given that $\mathrm{aSMA}^{+}$ mesenchymal cell populations and $\mathrm{Cdh} 5^{+}$endothelial cells in the bones showed minimal Colla1 expression (Fig. 1d). These results indicate that the contribution of $\alpha \mathrm{SMA}^{+}$cells and $\mathrm{Cdh} 5^{+}$cells to Coll production in the bones is not rate limiting. Although Colla1 ${ }^{\text {smaKO }}$ mice did not reveal spontaneous bone fractures, profound accumulation of aSMA-expressing cells in periosteal area was observed (Supplementary Fig. 9g) in the spontaneous bone lesions of Collal $1^{\mathrm{fspKO}}$ mice, consistent with previous studies showing the involvement in fracture repair. Future studies will hopefully unravel whether Colla $1^{\text {smaKO }}$ mice may exhibit any bone healing deficiency ${ }^{47-50}$.

\section{Discussion}

Type I collagen (Col1) is the most abundant protein in the human body, especially in the bones. Over 25,000 publication have examined the role of Col1 in different aspects of biology in health and disease, but it remained unknown until this study what cellular lineages contribute to the synthesis and deposition of Coll during embryogenesis and bone development ${ }^{3,4}$. Osteoblasts and fibroblasts, differentiated from mesenchymal stem/stromal cells (MSCs), are speculated to be the major source of Coll in various organs. However, the precise contributions of Coll by different cell lineages in the formation and development of bones remain unknown. In this regard, previous dataset ${ }^{27}$ coupled with current analysis show that Col1 is predominantly produced by $\mathrm{Fap}^{+}$and $\mathrm{Fsp}^{+}$mesenchymal cells in the bone. Based on this data, we generated novel genetically engineered mouse models (GEMMs) for the deletion of Coll specifically in $\mathrm{Fap}^{+}$or Fsp1 ${ }^{+}$cells. A Colla1 loxP/loxP mouse strain was generated and crossed with CMV-Cre, Fap-Cre, Fsp1 (S100a4)-Cre, Cdh5-Cre, and $\alpha S M A$ (Acta2)-Cre mice to probe the origin and function of Coll during embryogenesis and bone development.

Our results demonstrate that systemic (whole-body) deletion of Colla1 (exons 2-5 truncation) employing CMV-Cre mice (Colla1 ${ }^{\mathrm{cmvKO}}$ ) leads to arrested embryonic development and embryonic lethality around embryonic day 6-7 (E6-7), underscoring the essential role of Coll during gastrulation stage. Deletion of Col1 in $\mathrm{Fap}^{+}$cells leads to embryonic lethality at around E16 associated with several skeletal and vascular defects. This observation is in alliance with previous studies with Fap ${ }^{+}$ cells identified as mesenchymal cells surrounding the vasculature and in the skeletal structures 51,52 . These observations suggest the essential role of $\mathrm{Fap}^{+}$cells in the synthesis of Coll during the embryonic development of the bones. The observed phenotype of Colla $1^{\text {fapKO }}$ mice is analogous to the human type II Osteogenesis Imperfecta, which is lethal in the perinatal period due to critical genetic defects in COL1A1 or COL1A2 resulting in multiple rib and/or long bone fractures ${ }^{53-55}$.

Many different studies identified Fsp1 ${ }^{+}$cells in the developing skeletal tissue during embryogenesis ${ }^{35,56,57}$. Specific deletion of Col1 in $\mathrm{Fsp}^{+}$cells leads to an Osteogenesis Imperfecta-like phenotype and with most Collal $1^{\text {fspKO }}$ mice dying at the age of 5-12 months. The phenotype of Collal fspKO mice is comparable to the human type III Osteogenesis Imperfecta, and relevant mouse models previously reported $6,13-15,19,38,58-60$. While previous models of OI were based on systemic Col1 mutation and/or deletion, our studies identity the precise Fsp1 cell lineage that is responsible for Coll production in maturing bones starting about three weeks of age, and absence of such synthesis by the Fsp $1^{+}$cells leads to abnormal bones, resulting in easy fractures with minimal trauma. Our studies collectively suggest that early skeletal development during 
a

Col1a1spko

(Fsp1-Cre;Col1a1/oxP/loxP)

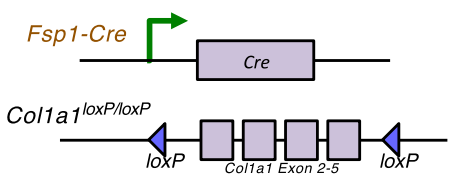

b $\quad-$ WT $\left(\right.$ Col1at $\left.{ }^{\text {loxPloxP }}\right) \mathrm{n}=24$

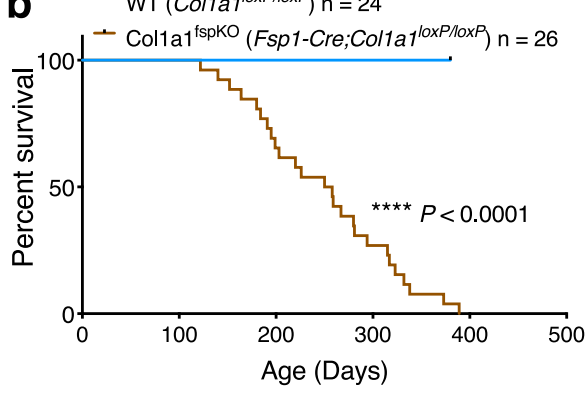

d Age: 1 month

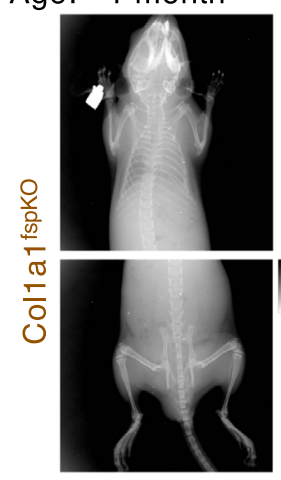

2 month

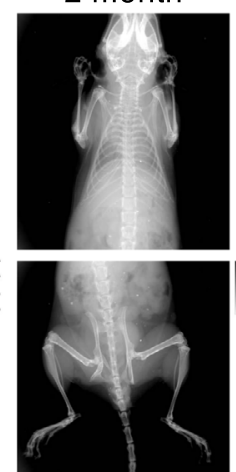

e

Phenotypic summary of Fsp1-Cre;Col1a1 10xPloxP (Col1a1 $1^{\text {tspko) }}$ mice $\mathbf{f}$

\begin{tabular}{|c|c|c|c|c|c|c|}
\hline Age (month) & $\begin{array}{c}1 \\
(n=8)\end{array}$ & $\begin{array}{c}2 \\
(n=8\end{array}$ & $\begin{array}{c}3 \\
n=8\end{array}$ & $\begin{array}{c}4 \\
(n=8)\end{array}$ & $\begin{array}{c}5 \\
(n=8)\end{array}$ & $\begin{array}{c}6 \\
(n=8)\end{array}$ \\
\hline Pelvis/Ischium & 0 & 3 & 6 & 7 & 8 & 8 \\
\hline $\begin{array}{l}\text { fracture } \\
\text { Elbow joint }\end{array}$ & & & & & & \\
\hline fractur & $(0 \%)$ & (0\%) & $(50 \%)$ & $(75 \%)$ & $(87.5 \%)$ & $(100 \%)$ \\
\hline
\end{tabular}

Phenotypic summary of Col1 1 1 10xP/loxP (WT) mice

\begin{tabular}{ccccccc}
\hline Age (month) & 1 & 2 & 3 & 4 & 5 & 6 \\
$(n=6)$ & $(n=6)$ & $(n=6)$ & $(n=6)$ & $(n=6)$ & $\begin{array}{c}6 \\
(n=6)\end{array}$ \\
\hline Pelvis/schium & 0 & 0 & 0 & 0 & 0 & 0
\end{tabular}

Pelvis/Ischim
fracture

fracture

fracture

g
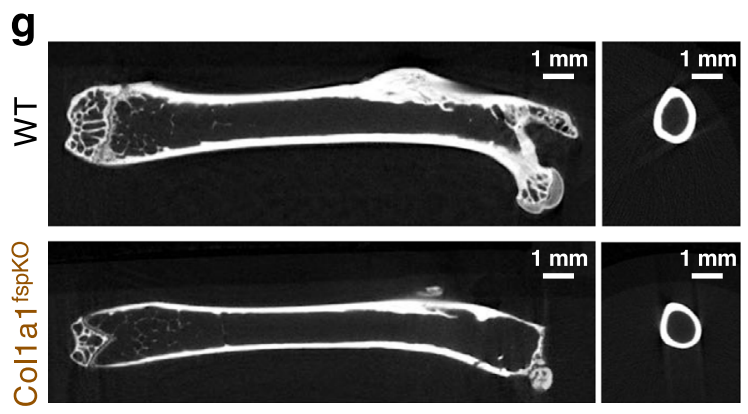

3 month

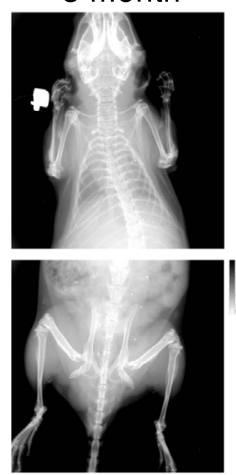

4 month

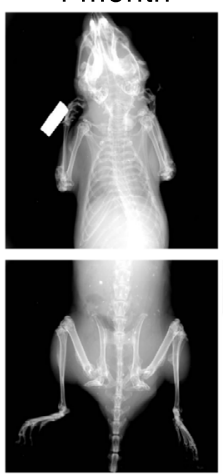

5 month

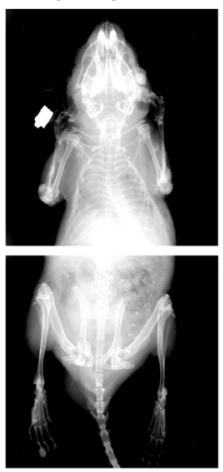

6 month
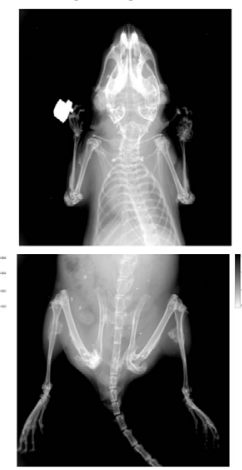
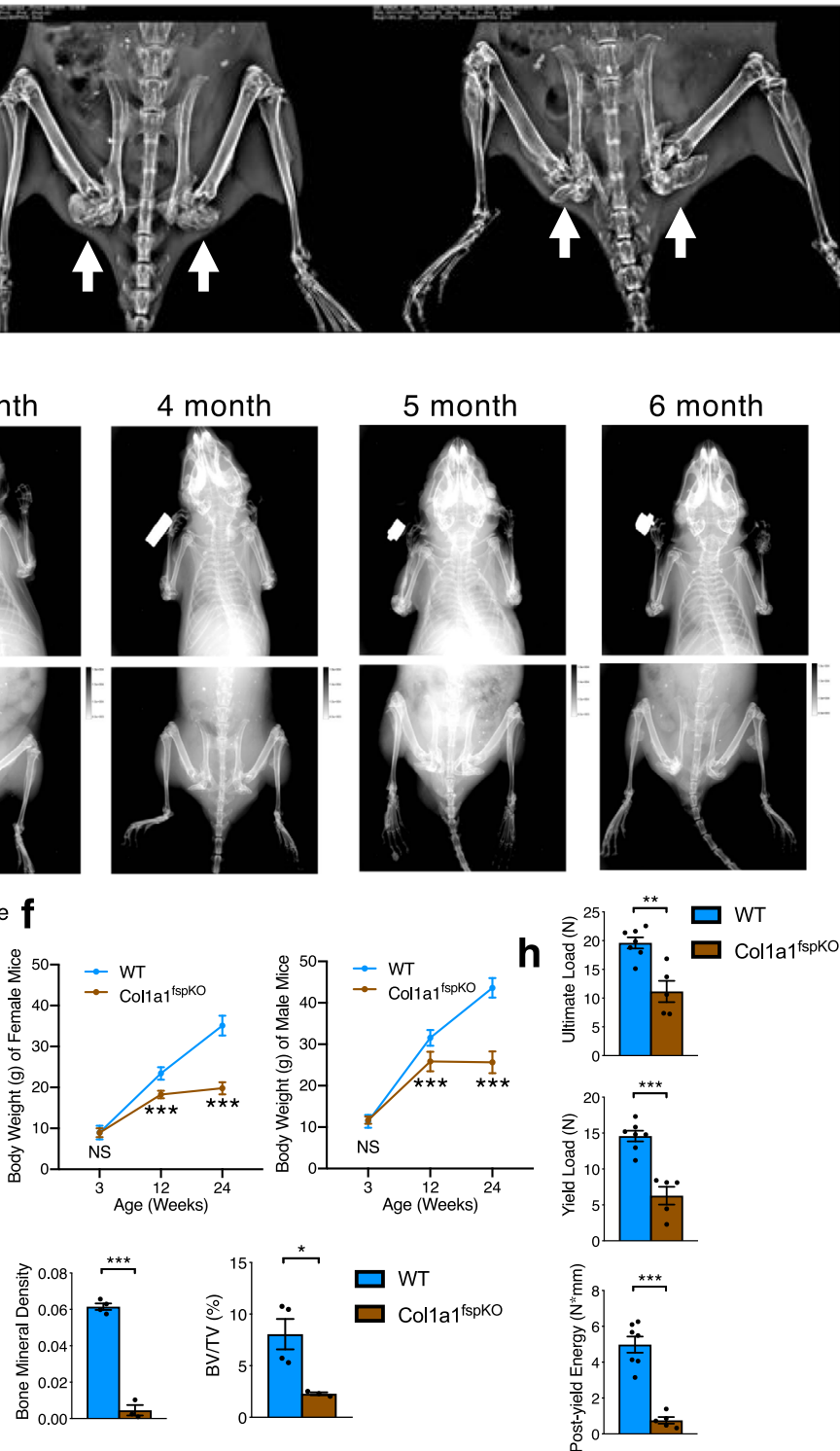
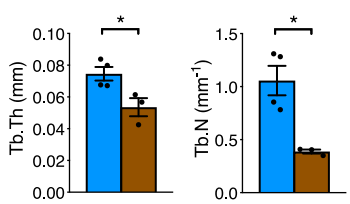

$\stackrel{\star \star *}{7} \square$ WT
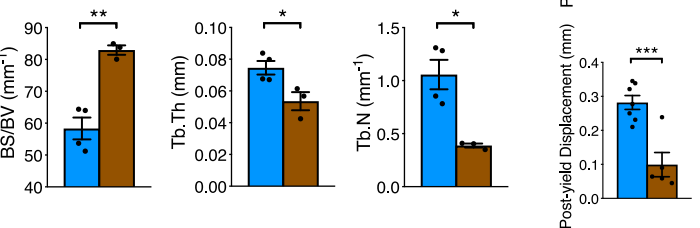

embryogenesis and the early postnatal period might rely on Fap ${ }^{+}$ cells for the production of Coll, and at a later stage the role of $\mathrm{Fsp}^{+}$cells becomes prominent. Deletion of Col1 from aSMA ${ }^{+}$ cells did not result in any adversity and mice were normal with expected lifespan. Same is true when Coll was deleted in Cdh5 ${ }^{+}$ endothelial cells (a common biomarker for most endothelial cells). This experimental arm served as an important control, because some reports suggest that angiogenic endothelial cells during embryogenesis and organogenesis are shown to produce type I collagen. All together, these experiments suggest that $\mathrm{aSMA}^{+}$ mesenchymal cells do not compensate for the loss of Coll from Fap or Fsp1 cell lineages. Moreover, the functions of Fap ${ }^{+}$cells and $\mathrm{Fsp}^{+}$cells are mutually exclusive in the context of embryogenesis and bone formation. 
Fig. 5 Specific deletion of Col1 in Fsp1-lineage cells causes Osteogenesis Imperfecta phenotype. a Genetic strategy to delete Colla1 specifically in Fsp1lineage cells by crossing the Colla 1oxP/loxP mice with Fsp1-Cre mice. b Survival of wild-type (WT; with genotype of Fsp1-Cre-negative;Colla1/oxP/loxP) and Colla1 fspKO (Fsp1-Cre;Col1a7loxP/loxP) mice $(n=24$ and 26, respectively). Kaplan-Meier plots and the log rank Mantel-Cox test were used to evaluate statistical differences of survival. ${ }^{\star \star \star \star} P<0.0001$. c Micro-CT scanning of 6 -month-old WT and Col1a1 ${ }^{\text {fspKO }}$ mice (with white arrows indicating the fractures in pelvis and ischium of Col1a $1^{\text {fspKO }}$ mice). d Radiograph of Col1a1 ${ }^{\text {fspKO }}$ mice at the age from 1 to 6 month. Radiograph of age-matched WT littermates was shown in Supplementary Fig. 7a. e Summary of the occurrence of fractures in pelvis, ischium, and/or joints, as examined by radiograph, of WT ( $n=6$ ) and Col1a1 ${ }^{f s p K O}(n=8)$ mice at the age from 1 to 6 month. f Body weight of WT and Colla1 $1^{f s p K O}$ mice $(n=6$ mice per group) at the age of 3,12 , and 24 month. The unpaired, two-tailed $t$ test was used to compare the mean of two independent groups. ${ }^{\star \star \star} P=9.57 \mathrm{E}-05$ (female 12 weeks), $7.15 \mathrm{E}-07$ (female 24 weeks), 0.00096 (male 12 weeks), 2.35E-07 (male 24 weeks). Data are represented as mean \pm SEM. $\mathbf{g}$ The micro-CT images of trabecular and cortical bones of femurs from 6-month-old WT mice $(n=4)$ and Colla1 ${ }^{f s p K O}$ mice $(n=3)$. The trabecular bone material property measurements are compared as bone mineral density $\left(B M D ;{ }^{* \star} P=1.04 \mathrm{E}-05\right)$, bone volume/total volume (BV/TV; $\left.{ }^{\star} P=0.0289\right)$, bone surface area/bone volume (BS/BV; $\left.{ }^{\star}{ }^{\star} P=0.00212\right)$, trabecular thickness (Tb.Th; ${ }^{\star} P=0.0287$ ), and trabecular number (Tb.N; ${ }^{\star} P=0.0160$ ). The unpaired, two-tailed $t$ test was used to compare the mean of two independent groups. Data are represented as mean \pm SEM. The cortical bone material property measurements are continued in Supplementary Fig. 7d. h Biomechanical (three-point bending) test results of femurs from 6-month-old WT mice $(n=7)$ and Col1a1 ${ }^{\text {fspKO }}$ mice $(n=5)$ were presented as ultimate load ( $\left.{ }^{\star \star} P=0.00135\right)$, yield load $\left.{ }^{\star \star \star} P=0.00012\right)$, post-yield energy $\left({ }^{\star \star \star} P=2.01 \mathrm{E}-05\right)$, and post-yield displacement $\left.{ }^{\star \star \star} P=0.00077\right)$. The unpaired, two-tailed $t$ test was used to compare the mean of two independent groups. Data are represented as mean \pm SEM.

These results also emphasize the crucial contribution of Coll specifically produced by $\mathrm{Fsp}^{+}{ }^{+}$osteoblasts and fibroblasts (in bone marrow and periosteum) to the structural maintenance and repair of the bones, which is consistent with previous notion that fibroblasts appear at fracture site and are responsible for collagen production/crosslinking upon encountering hematoma ${ }^{61}$. Our results also suggest that the Coll deposited by fibroblasts of Fsp1-lineage is essential for the formation of hematoma, which further impacts the subsequent steps leading to the repair of fractures including osteoblast differentiation and bone formation in the fractured callus ${ }^{62-64}$. The identification of the Fsp $1^{+}$cells as critical and rate-limiting source of Col1 in developing bone and adult bone undergoing repair and regeneration provides new insights into potential cell-based therapy for Osteogenesis Imperfecta. Future studies may examine whether $\mathrm{Fsp}^{+}$cells may provide cell-based therapy opportunities for Osteogenesis Imperfecta via transplantation.

Taken together, our results demonstrate that Coll produced by $\mathrm{Fap}^{+}$cells is essential for the embryonic development of skeletal system, while Coll produced by $\mathrm{Fsp}^{+}$cells is critical for the postnatal maintenance and repair of the bones. Utilizing multiple transgenic mouse models and single-cell RNA-sequencing analyses, this study for the first time provided comprehensive investigations on the functional roles of Coll with respect to its specific cellular origin during embryogenesis, osteogenesis, and bone pathogenesis.

\section{Methods}

Mice. All mice were housed under standard housing conditions at MDACC animal facilities, and all animal procedures were reviewed and approved by the MDACC Institutional Animal Care and Use Committee.

Fsp1-Cre $e^{65,66}$ and $\alpha S M A-C r e^{67}$ mouse strains were previously documented. Cdh5Cre (017968; B6.129-Tg-Cdh5-cre-1Spe/J), CMV-Cre (006054; B6.C-Tg-CMV-cre$1 \mathrm{Cgn} / \mathrm{J})$, and LSL-YFP (006148; B6.129 × 1-Gt-ROSA-26Sortm1-EYFP-Cos/J) mouse strains were purchased from Jackson Laboratory. Osteogenesis imperfecta murine (OIM) strain harboring Col1a2 mutation (001815; B6C3Fe a/a-Colla2oim/J) was purchased from Jackson Laboratory. This Colla2 spontaneous mutation is a single nucleotide deletion $(\mathrm{G})$ that alters the terminal approximately 50 amino acids of the pro-alpha $2 \mathrm{C}$-propeptide and prevents association with the pro-alphal chains. Colla1 loxP/loxP mouse strain (with loxP-flanked exons 2-5) was established in the Genetically Engineered Mouse Facility at MD Anderson Cancer Center (MDACC)

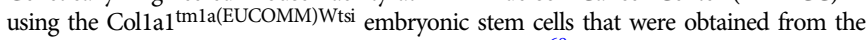
European Mouse Mutant Cell Repository (EuMMCR) ${ }^{6}$. Both female and male mice with desired genotype(s) were used for experimental mice.

Genotyping. Tail DNA was used for genotyping PCR analysis of animals and embryos older than E14.5. Yolk sac DNA was used for genotyping PCR of embryos at earlier stages. Recombination PCR of Colla1 ${ }^{\text {loxP } / l o x P}$ allele on DNA from multiple major organs of all experimental mice was routinely performed to exclude the possibility of germline recombination.
Histology and immunohistochemistry. For paraffin-fixed samples, mouse tissues were fixed in $10 \%$ neutral buffered formalin, embedded in paraffin, and sectioned at $5 \mu \mathrm{m}$ thickness. Sections were processed for hematoxylin and eosin (H\&E) staining. Masson's trichrome stain (MTS) was conducted using Gomori's Trichrome Stain Kit (38016SS2, Leica Biosystems). Picrosirius red staining for collagen was conducted using 0.1\% Picrosirius Red (Direct Red80; Sigma-Aldrich) and counterstained with Weigert's haematoxylin. Images were captured with a Leica DM 1000 LED microscope and an MC120 HD Microscope Camera with LAS V4.4 Software (Leica). Safranin-O/Fast Green staining was conducted using $0.1 \%$ Safranin-O solution and $0.1 \%$ Fast Green solution (Electron Microscopy Sciences).

Formalin-fixed, paraffin-embedded sections were processed for immunohistochemical staining as previously documented ${ }^{69,70}$. Sections were incubated with primary antibody against type I collagen (Col1; SouthernBiotech, 1310-01, lot\#B2918-XB89, 1:200), followed by biotinylated donkey anti-goat secondary antibody (Jackson ImmunoResearch, 705-066-147, lot\#127411, 1:400), and streptavidin HRP (Biocare Medical). For all immunolabeling experiments, sections were developed by DAB and counterstained with hematoxylin. Immunofluorescence staining on formalin-fixed, paraffin-embedded sections was conducted using the following primary antibodies: rabbit anti-GFP/YFP (Abcam, ab290, lot\#GR3321614-1, 1:500), chicken anti-GFP/YFP (Abcam, ab13970, lot\# GR3190550-13, 1:400), rabbit anti-osteocalcin/OCN (Millipore/Sigma-Aldrich, ab10911, lot\#3670782, 1:200), rat anti-Ly6G (Abcam, ab25377, clone RB6-8C5, lot\#GR3391587-2, 1:100), and mouse anti-RFP/tdTomato (Thermo Fisher, MA515257, clone RF5R, lot\#SD247965, 1:100). The staining was then followed by AF488/594-labeled secondary antibodies: Thermo Fisher A11012 (lot\#1933366), A11039 (lot\#2079383), A11008 (lot\#1937184), and A21203 (lot\#1722995), all in 1:400 dilution. Slides were then mounted with DAPI-containing Vectashield Mounting Medium (Vector Laboratories), visualized under the LSM800 confocal laser scanning microscope, and analyzed with ZEN software version 2.1 (Zeiss).

Radiography (X-ray) and micro computed tomography (micro-CT) Scanning Imaging operations were conducted at MDACC Small Animal Imaging Facility. Radiography of mice was conducted using the Bruker Xtreme system. Micro-CT scanning was performed using the Bruker SkyScan 1276 micro-CT system (Bruker BioSpin Corporation). The scanning parameters are: resolution: $13 \mu \mathrm{m}$; pixel size: $13.083 \mu \mathrm{m}$; voltage: $55 \mathrm{kV}$; current: $200 \mu \mathrm{A}$; rotation step (deg): 0.400; duration: $11 \mathrm{~min}$; exposure time: $500 \mathrm{~ms}$; filter: $0.25 \mathrm{~mm} \mathrm{Al}$; matrix: $2016 \times 1344$. The femurs of 6-month-old Collal ${ }^{\text {fspKO }}$ mice and wild-type (WT) littermates were scanned and measured for the bone material properties. The trabecular bone parameters were measured in the distal metaphysis of the femurs, while the cortical bone parameters were measured at the femur mid-diaphysis. The detected bone parameters included trabecular or cortical bone mineral density (BMD), bone volume/ total volume (BV/TV), trabecular or cortical bone surface area/bone volume (BS/ $\mathrm{BV}$ ), trabecular thickness (Tb.Th), trabecular number (Tb.N), cortical bone total area (Tt.Ar), cortical bone area (Ct.Ar), cortical bone marrow area (Ma.Ar),), cortical bone area/total area (Ct.Ar/Tt.Ar), and cortical thickness (Ct.Th). The analysis was conducted using Bruker SkyScan DataViewer software version 1.5.0.

Biomechanical (three-point bending) examination. The femurs from Collal ${ }^{\text {fspKO }}$ mice and WT littermates of 6-month age were freshly collected and tested at room temperature using an Instron 5848 microtester (Instron Inc.), as previously documented ${ }^{17}$. The femurs were examined to failure in three-point bending test at a rate of $0.1 \mathrm{~mm} / \mathrm{s}$. A $100 \mathrm{~N}$ load cell was used to collect data. Load and displacement data were captured at a rate of $40 \mathrm{~Hz}$ by Bluehill Software version 2.9 (Instron). The span length used in the examination was $7.3 \mathrm{~mm}$. The maximum load was determined by the highest load value recorded before the specimen fractured. 
a

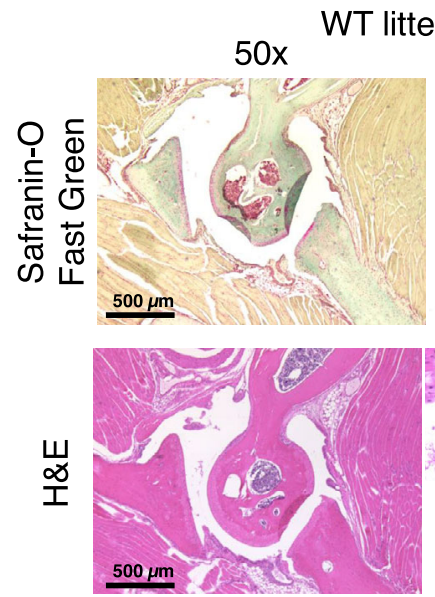

b



$200 x$
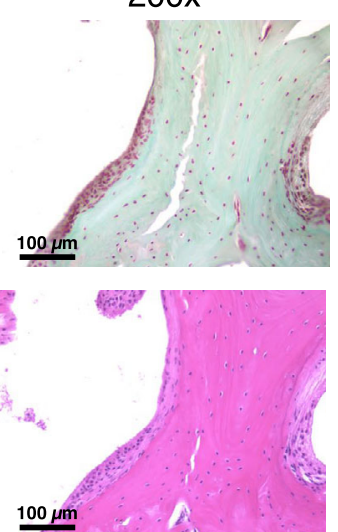

Col1a1 ${ }^{\text {fspko }}$
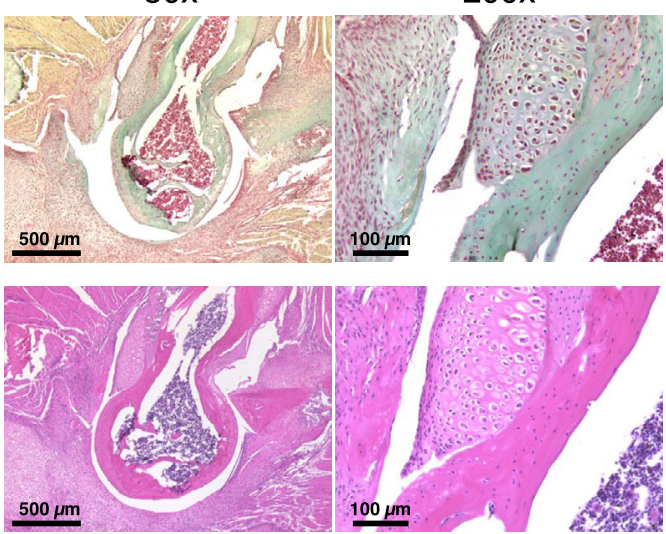

C

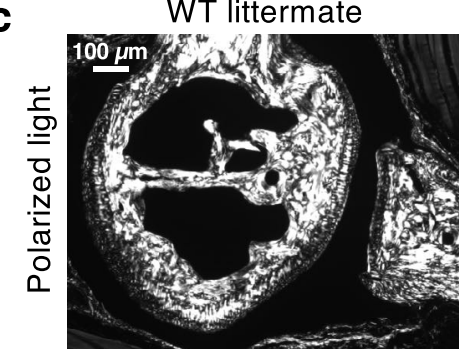

Col1a1 ${ }^{\text {fspKo }}$

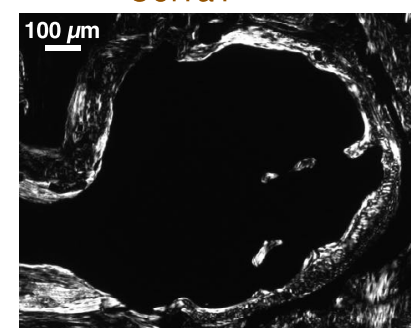

d
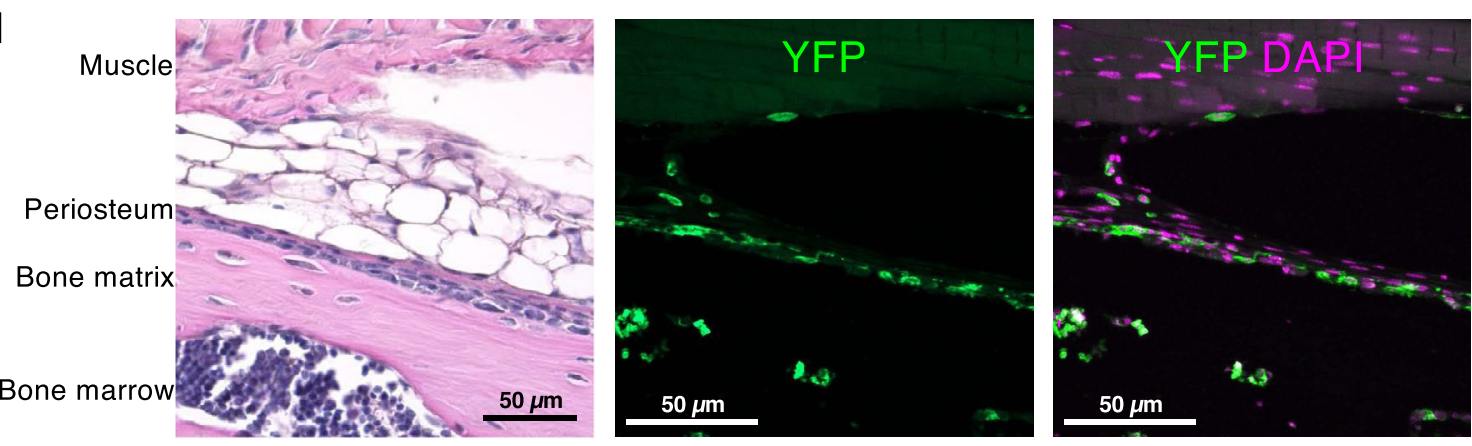

e
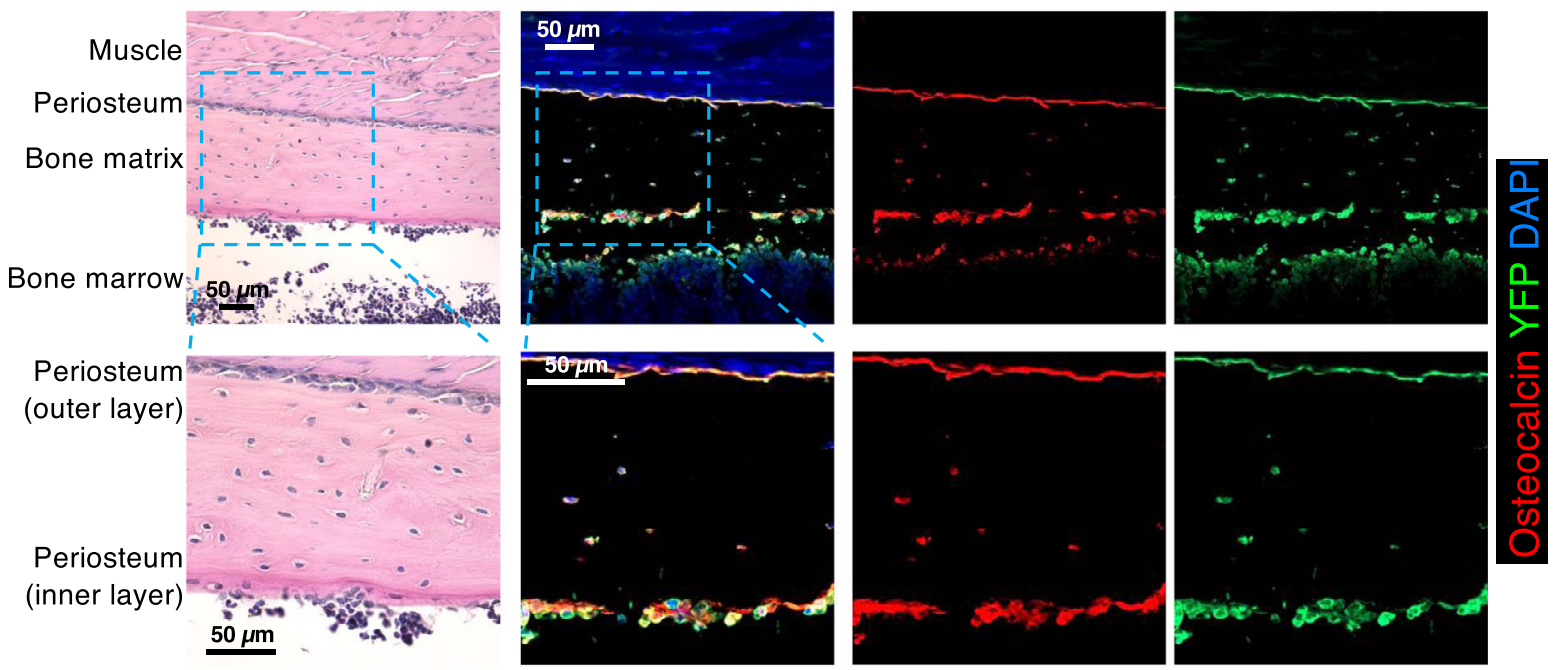

Fig. 6 Histological analysis of bone tissues from Col1a1fspKO mice and Fsp1-Cre;LSL-YFP mice. a, b H\&E staining and Safranin-O/Fast Green staining of the forelimb elbow joints of 6-month-old WT and Colla1 ${ }^{15 p K O}$ mice (a). Quantification of \% positive area for Safranin-O/Fast Green staining was based on three mice per group (b). The unpaired, two-tailed $t$ test was used to compare the mean of two independent groups. ${ }^{\star \star} P=0.00946$. Data are represented as mean $\pm S E M$.

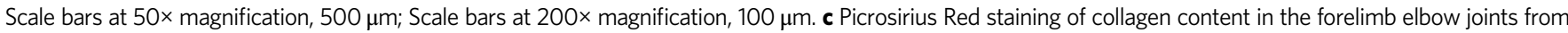
six-month-old WT and CollaffspKO mice ( $n=3$ mice per group) examined by circularly polarized light microscopy. Scale bars, $100 \mu \mathrm{m}$. $\mathbf{d}$ Representative immunofluorescence images of humerus serial sections from lineage-tracing Fsp1-Cre;LSL-YFP mice ( $n=3$ mice at two-month-old) stained for Fsp1-Cre-induced YFP. Scale bars, $50 \mu \mathrm{m}$. e Representative H\&E and immunofluorescence images of humerus serial sections from Fsp1-Cre;LSL-YFP mice ( $n=3$ mice at two-monthold) stained for osteoblast marker osteocalcin (red) and Fsp1-Cre-induced YFP (green). Scale bars, $50 \mu \mathrm{m}$. 

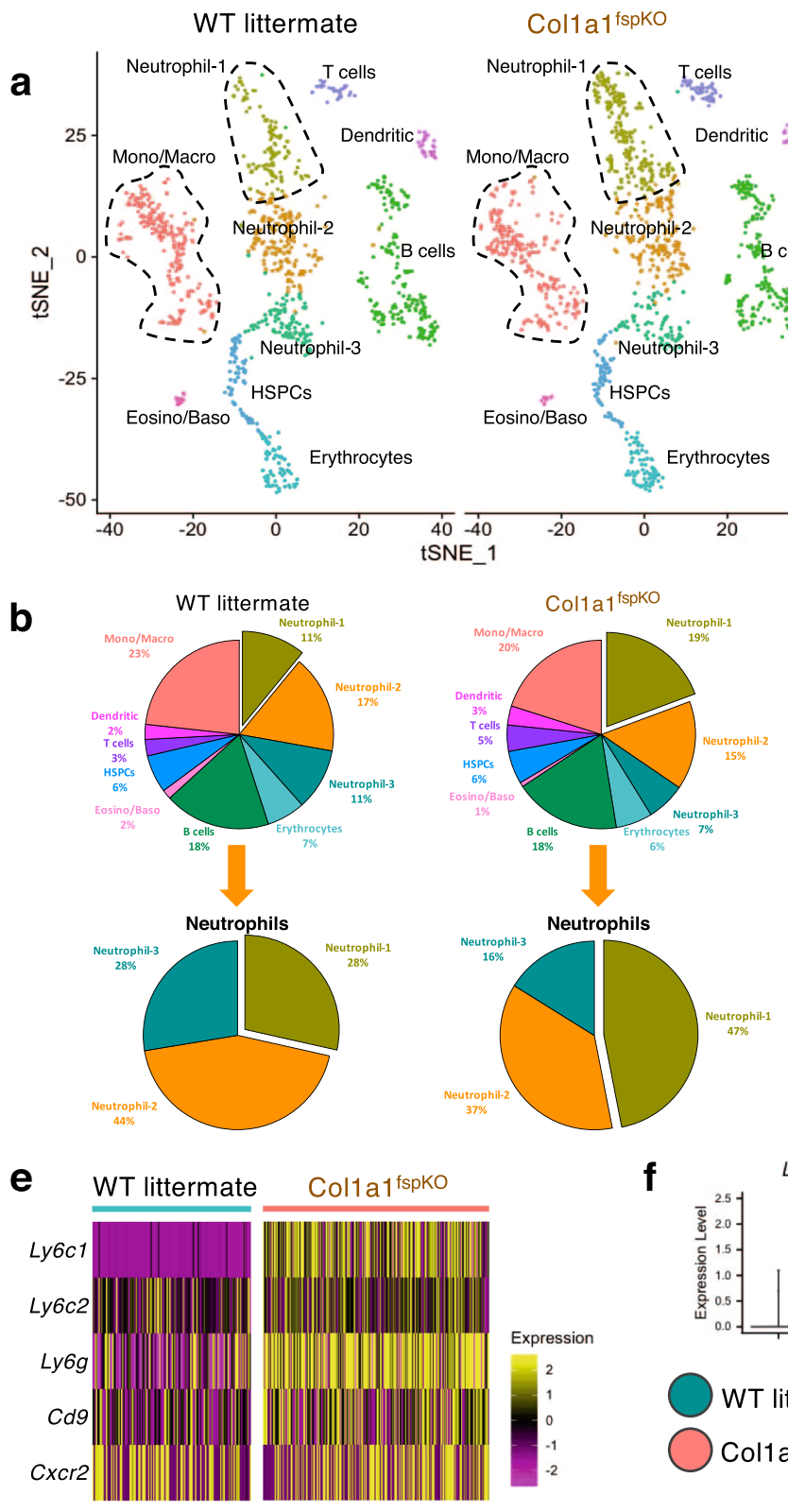

f
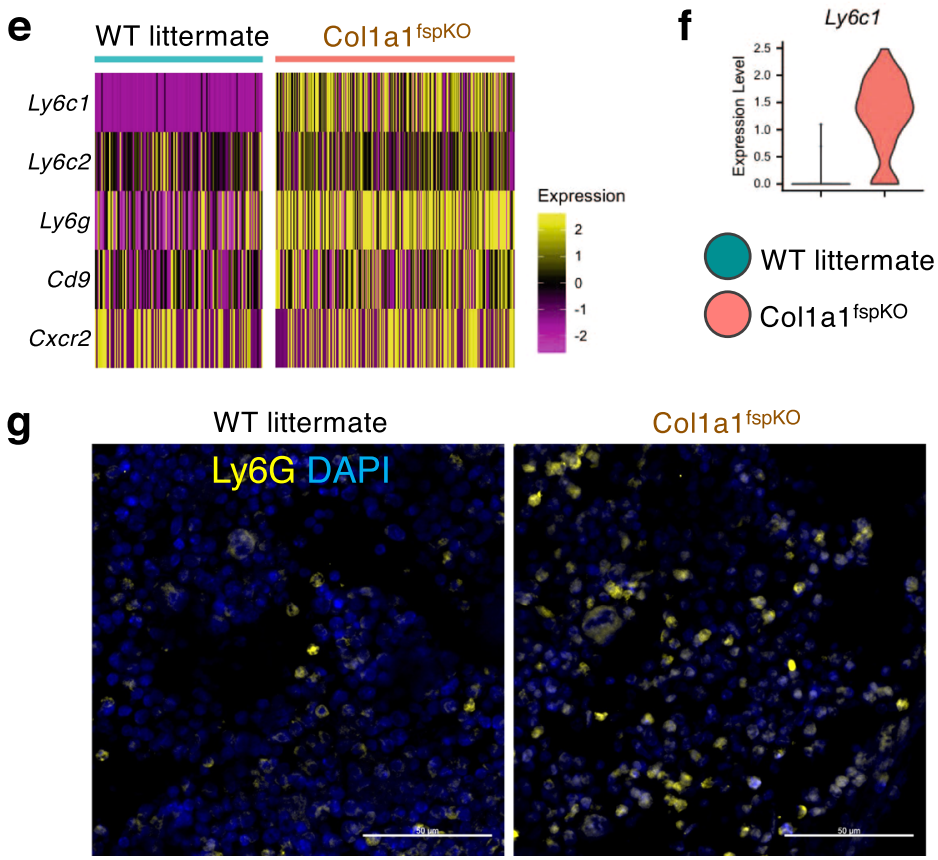

Col1a $1^{\text {fspko }}$
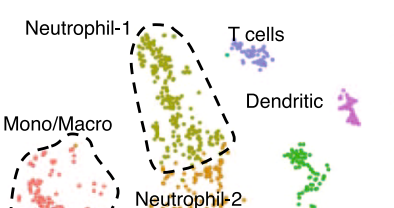

B cells

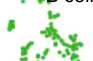

की

Mono/Macro

Neutrophil-1

Neutrophil-2

- Neutrophil-3

- B cells

- Erythrocytes

HSPCs

- T cells

- Eosino/Baso

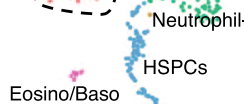

Erythrocytes

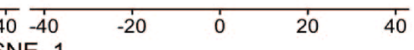

Col1 a $1^{\text {fspko }}$

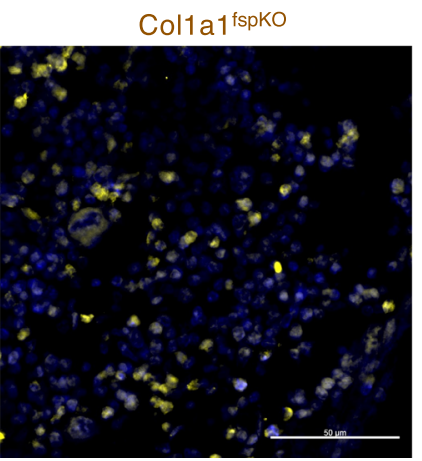

Top gene signature of Neutrophil-1 cluster
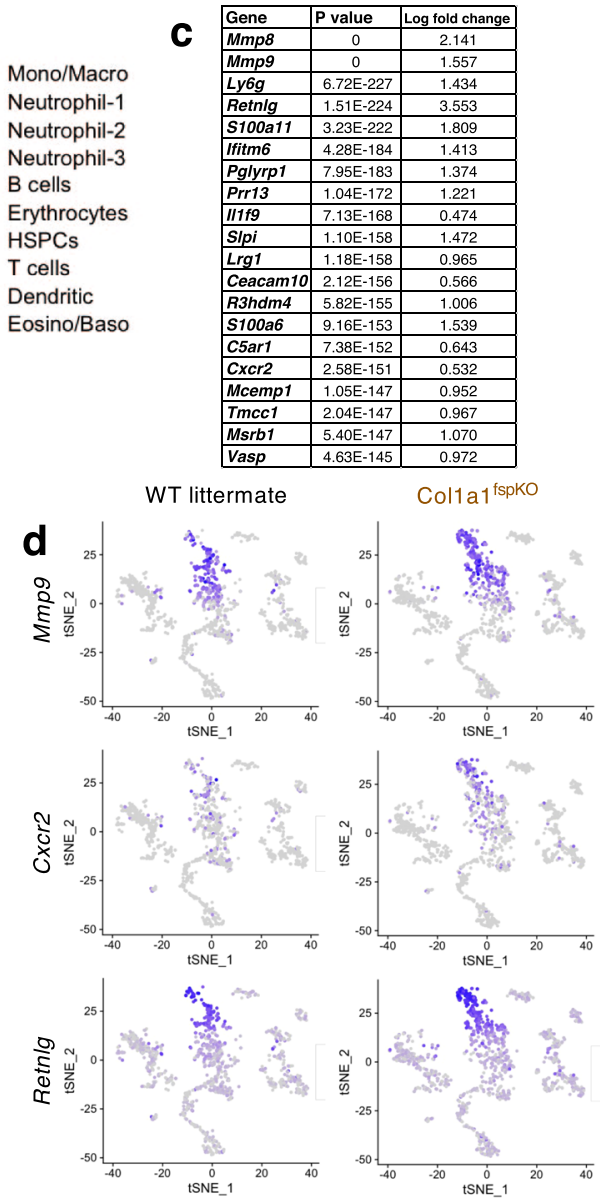

Ly6c2

Ly6g
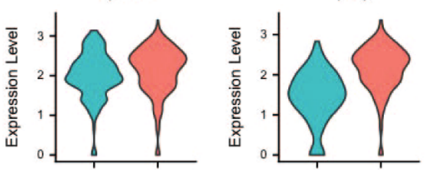

$C d 9$
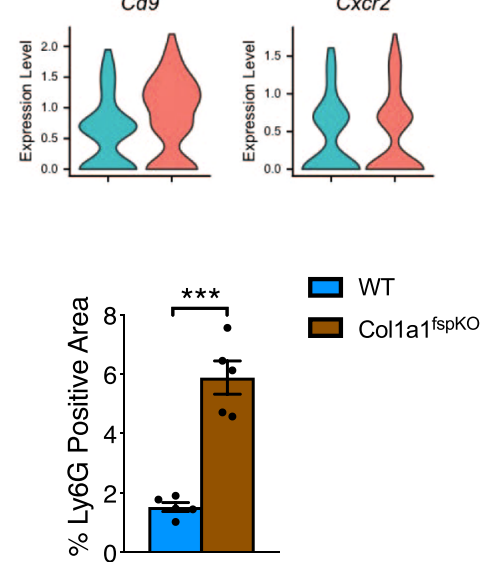

Single-cell RNA-sequencing (sc-RNA-seq). For the sc-RNA-seq on bone marrow cells conducted in this study, femur and tibia bones of Collal ${ }^{\text {fspKO }}$ mice (and agematched wild-type littermate; $n=2$ mice per group) were collected and flushed to obtain bone marrow fractions. Bone marrow cells, pooled for each group, were filtered by $70 \mu \mathrm{m}$ cell strainers, and resuspended in PBS/2\%FBS as single cell suspension. The single cell suspension was stained with Live/Dead viability dye eFluor 780 (65-0865-14, eBioscience), filtered through a $40 \mu \mathrm{m}$ mesh, and then sorted for live cells with Aria II sorter (BD Biosciences) at the South Campus Flow Cytometry Core Laboratory of
MDACC. These samples were examined by sc-RNA-seq at the Sequencing and Microarray Facility of MDACC. Single cell Gel Bead-In-Emulsions (GEMs) generation and barcoding, post GEM-RT cleanup and cDNA amplification, library construction and Illumina-ready sequencing library generation were prepared by following the manufacturer's guidelines. High Sensitivity dsDNA Qubit kit was used to estimate the cDNA and Library concentration. HS DNA Bioanalyzer was used for the quantification of cDNA. DNA 1000 Bioanalyzer was used for the quantification of libraries. The "cloupe" files were generated by using Cell Ranger software pipelines following 
Fig. 7 Single-cell RNA-sequencing analysis of bone marrow fractions from Col1a1fspKO mice. a-c Single-cell RNA-sequencing analysis of cell mixture of bone marrow fractions from 2-month-old WT and Col1a $1^{f s p K O}$ mice $(n=2$ per group). Functional clusters of cells were defined with group definition listed, as implemented in the Seurat R package (a). Mono/Macro: monocyte/macrophage cluster. HSPCs: hematopoietic stem and progenitor cells. Dendritic: dendritic cell cluster. Eosino/Baso: eosinophil/basophil cluster. $\mathbf{b}$ Pie charts showing the percentage of each of the functional cell clusters in bone marrow fractions from WT and Col1a1 $1^{\text {fspKO }}$ mice. Total neutrophil clusters containing 3 subpopulations (1-3) were further plotted as pie charts. (c) List of top signature genes of "Neutrophil-1" cluster shown in (a) and (b), presented with $P$ value (based on the non-parameteric Wilcoxon Rank Sum test) and Log2 fold change. Continued in Supplementary Fig. 8a-c. d Expression profile of Mmp9, Cxcl12, and Retnlg among defined cell clusters in the bone marrow fractions from WT and Col1a $1^{\text {fspKO }}$ mice shown in UMAP plot. Continued in Supplementary Fig. 8d. e, $\mathbf{f}$ Top upregulated genes of Neutrophil-1 cluster in Col1a1fspKO mice than WT mice shown in heat map plot (e) or violin plot (f). $\mathbf{g}$ Representative immunofluorescence images and quantification results of humerus bone marrow from two-month-old WT and Col1a1 $1^{\mathrm{fspKO}}$ mice $(n=5$ per group) stained for Neutrophil-1 marker Ly6G (yellow). Scale bars, $50 \mu \mathrm{m}$. The unpaired, two-tailed $t$ test was used to compare the mean of two independent groups. ${ }^{\star \star \star} P=0.00096$. Data are represented as mean \pm SEM.

manufacturer's guidelines. Cells from unfractionated tumor were encapsulated using 10X Genomics' Chromium controller and Single Cell 3' Reagent Kits v2. Following capture and lysis, cDNA was synthesized and amplified to construct Illumina sequencing libraries. The libraries from about 1,000 cells per sample were sequenced with Illumina Nextseq 500. The run format was 26 cycles for read 1, 8 cycles index 1 , and 124 cycles for read 2. sc-RNA-seq data was processed by the Sequencing and Microarray Facility in MD Anderson Cancer Center. Library Seurat (version 3.6.1), dplyr (version 1.0.7) and cowplot (version 1.1.1) were loaded into R (version 3.6.1) to explore QC metrics, filter cells, normalize data, cluster cells, and identify cluster biomarkers. To filter out low-quality cells, a threshold with a minimum of 200 and a maximum of 7000 genes per cell was used. Cells with more than $10 \%$ of the mitochondrial genome were also removed for further analysis. "RunTSNE" function was used for clustering the cells. Based on the "JackStrawPlot" and "ElbowPlot" functions, the first 27 principal components were used for TSNE projection and clustering analysis. "FindAllMarkers" function was used to identify the specific markers for each cell cluster. "DoHeatmap" function was used to show the top 10 genes in each cluster. "VlnPlot" function was used to show expression probability distributions across cell clusters of the genes we selected to assign the cell type identity, and the genes that we were interested in.

The previous published single-cell RNA-sequencing (sc-RNA-seq) dataset on bone and bone marrow cell populations of healthy mice ${ }^{27}$ were retrieved from the Gene Expression Omnibus (GEO) repository (GSE128423). As previously described ${ }^{27}$, femur and tibia bones of healthy C57BL/6 mice were collected and separated into bone and bone marrow fractions. Stromal cells from the bone marrow were isolated after STEMxyme1/DispaseII digestion. Stromal cells from the bone fraction were isolated after STEMxyme1/DispaseII digestion of the cut/crushed bone fragments. Cell mixtures were then filtered through $70 \mu \mathrm{m}$ cell strainers, lysed with ACK-lysis buffer, and prepared for FACS cell sorting. Bone marrow stromal cells were enriched by sorting of live cells that are negative for erythroid markers (CD71/Ter119) and immune lineage markers (CD45/CD3/B220/CD19/Gr-1/CD11b). This dataset was analyzed using a similar methodology as the above sc-RNA-seq data generated in this study. A total of 12530 cells from bone fraction and 14875 cells from bone marrow fraction were analyzed. Functional clusters of cells were defined with indicated group definition, as implemented in the Seurat R package. "RunUMAP" function was used for clustering the cells. "FindAllMarkers" function was used to identify the specific markers for each cell cluster. "FindMarkers" function was used to compare the differentially expressed marker genes for indicated cell cluster between control and knockout groups.

"DoHeatmap" function was used to show the top 10 genes in each cluster. "VlnPlot" function was used to show expression probability distributions across cell clusters of the genes we selected to assign the cell type identity, and the genes that we were interested in.

Statistics. Statistical analyses were performed with unpaired, two-tailed $t$ test, or one-way ANOVA with Tukey's multiple comparison test using GraphPad Prism version 8.0.0 (GraphPad Software, San Diego, CA, USA). Kaplan-Meier plots were drawn for survival analysis and the log rank Mantel-Cox test was used to evaluate statistical differences. Data met the assumptions of each statistical test, where variance was not equal (determined by an $F$-test) Welch's correction for unequal variances was applied. A $P$ value $<0.05$ was considered statistically significant. ${ }^{*} P<0.05,{ }^{* *} P<0.01,{ }^{* * *} P<0.001,{ }^{* * * *} P<0.0001$, NS not significant. Error bars represented standard error of the mean (S.E.M.) when multiple visual fields were averaged to produce a single value for each animal, which was then averaged again to represent the mean bar for the group in each graph.

Reporting summary. Further information on research design is available in the Nature Research Reporting Summary linked to this article.

\section{Data availability}

The authors declare that all data supporting the findings of this study are available within the paper and the supplementary information. The single-cell RNA-sequencing source data generated by this study have been deposited in Gene Expression Omnibus (GEO) database with the accession number GSE184360. The single-cell RNA-sequencing source data of a previously published dataset ${ }^{27}$ have been deposited in Gene Expression Omnibus (GEO) database with the accession number GEO GSE128423. Source data are provided with this paper.

Received: 3 February 2021; Accepted: 16 November 2021; Published online: 10 December 2021

\section{References}

1. Hay, E. D. Extracellular matrix. J. cell Biol. 91, 205s-223s (1981).

2. Lohler, J., Timpl, R. \& Jaenisch, R. Embryonic lethal mutation in mouse collagen I gene causes rupture of blood vessels and is associated with erythropoietic and mesenchymal cell death. Cell 38, 597-607 (1984).

3. Zaidi, M. Skeletal remodeling in health and disease. Nat. Med. 13, 791-801 (2007)

4. Marini, J. C. et al. Osteogenesis imperfecta. Nat. Rev. Dis. Prim. 3, 17052 (2017)

5. Rauch, F. \& Glorieux, F. H. Osteogenesis imperfecta. Lancet 363, 1377-1385 (2004).

6. Chipman, S. D. et al. Defective pro alpha 2(I) collagen synthesis in a recessive mutation in mice: a model of human osteogenesis imperfecta. Proc. Natl Acad. Sci. USA 90, 1701-1705 (1993).

7. Kuznetsova, N., McBride, D. J. Jr. \& Leikin, S. Osteogenesis imperfecta murine: interaction between type I collagen homotrimers. J. Mol. Biol. 309, 807-815 (2001).

8. Miles, C. A., Sims, T. J., Camacho, N. P. \& Bailey, A. J. The role of the alpha2 chain in the stabilization of the collagen type I heterotrimer: a study of the type I homotrimer in oim mouse tissues. J. Mol. Biol. 321, 797-805 (2002).

9. Kuznetsova, N. V., McBride, D. J. \& Leikin, S. Changes in thermal stability and microunfolding pattern of collagen helix resulting from the loss of alpha2(I) chain in osteogenesis imperfecta murine. J. Mol. Biol. 331, 191-200 (2003).

10. Kuznetsova, N. V., Forlino, A., Cabral, W. A., Marini, J. C. \& Leikin, S. Structure, stability and interactions of type I collagen with GLY349-CYS substitution in alpha 1(I) chain in a murine Osteogenesis Imperfecta model. Matrix Biol.: J. Int. Soc. Matrix Biol. 23, 101-112 (2004).

11. Schnieke, A., Harbers, K. \& Jaenisch, R. Embryonic lethal mutation in mice induced by retrovirus insertion into the alpha (I) collagen gene. Nature 304, 315-320 (1983).

12. Bonadio, J. et al. A murine skeletal adaptation that significantly increases cortical bone mechanical properties. Implications for human skeletal fragility. J. Clin. Investig. 92, 1697-1705 (1993).

13. Lisse, T. S. et al. ER stress-mediated apoptosis in a new mouse model of osteogenesis imperfecta. PLoS Genet 4, e7 (2008).

14. Chen, F. et al. First mouse model for combined osteogenesis imperfecta and Ehlers-Danlos syndrome. J. Bone Min. Res. 29, 1412-1423 (2014).

15. Besio, R. et al. Early fracture healing is delayed in the Col1a2(+/G610C) osteogenesis imperfecta murine model. Calcif. Tissue Int 103, 653-662 (2018).

16. Daley, E. et al. Variable bone fragility associated with an Amish COL1A2 variant and a knock-in mouse model. J. Bone Min. Res. 25, 247-261 (2010).

17. Zieba J., et al. Fracture healing in collagen-related preclinical models of osteogenesis imperfecta. J Bone Miner Res. 35, 1132-1148 (2020).

18. Morello, R. et al. CRTAP is required for prolyl 3- hydroxylation and mutations cause recessive osteogenesis imperfecta. Cell 127, 291-304 (2006).

19. Liu, Y. et al. A novel transgenic murine model with persistently brittle bones simulating osteogenesis imperfecta type I. Bone 127, 646-655 (2019).

20. Florencio-Silva, R., Sasso, G. R., Sasso-Cerri, E., Simoes, M. J. \& Cerri, P. S. Biology of bone tissue: structure, function, and factors that influence bone cells. Biomed. Res Int. 2015, 421746 (2015).

21. Friedenstein, A. J., Chailakhyan, R. K., Latsinik, N. V., Panasyuk, A. F. \& Keiliss-Borok, I. V. Stromal cells responsible for transferring the 
microenvironment of the hemopoietic tissues. Cloning in vitro and retransplantation in vivo. Transplantation 17, 331-340 (1974).

22. Pittenger, M. F. et al. Multilineage potential of adult human mesenchymal stem cells. Science 284, 143-147 (1999).

23. Nakahara, H., Goldberg, V. M. \& Caplan, A. I. Culture-expanded human periosteal-derived cells exhibit osteochondral potential in vivo. J. Orthop. Res. 9, 465-476 (1991).

24. Friedenstein, A. J., Chailakhyan, R. K. \& Gerasimov, U. V. Bone marrow osteogenic stem cells: in vitro cultivation and transplantation in diffusion chambers. Cell Tissue Kinet. 20, 263-272 (1987).

25. Friedenstein, A. J., Chailakhjan, R. K. \& Lalykina, K. S. The development of fibroblast colonies in monolayer cultures of guinea-pig bone marrow and spleen cells. Cell Tissue Kinet. 3, 393-403 (1970).

26. Bohm, A. M. et al. Activation of skeletal stem and progenitor cells for bone regeneration is driven by PDGFRbeta signaling. Developmental cell 51, 236-254 e212 (2019)

27. Baryawno, N. et al. A cellular taxonomy of the bone marrow stroma in homeostasis and leukemia. Cell 177, 1915-1932 e1916 (2019).

28. Baccin, C. et al. Combined single-cell and spatial transcriptomics reveal the molecular, cellular and spatial bone marrow niche organization. Nat. cell Biol. 22, 38-48 (2020).

29. Ding, L. \& Morrison, S. J. Haematopoietic stem cells and early lymphoid progenitors occupy distinct bone marrow niches. Nature 495, 231-235 (2013).

30. Greenbaum, A. et al. CXCL12 in early mesenchymal progenitors is required for haematopoietic stem-cell maintenance. Nature 495, 227-230 (2013).

31. Delmas, P. D., Demiaux, B., Malaval, L., Chapuy, M. C. \& Meunier, P. J. [Osteocalcin (or bone gla-protein), a new biological marker for studying bone pathology]. Presse Med 15, 643-646 (1986).

32. Huang, W., Yang, S., Shao, J. \& Li, Y. P. Signaling and transcriptional regulation in osteoblast commitment and differentiation. Front. Biosci.: a J. virtual Libr. 12, 3068-3092 (2007)

33. Doege, K. J., Sasaki, M., Kimura, T. \& Yamada, Y. Complete coding sequence and deduced primary structure of the human cartilage large aggregating proteoglycan, aggrecan. Human-specific repeats, and additional alternatively spliced forms. J. Biol. Chem. 266, 894-902 (1991).

34. Chan, D., Cole, W. G., Chow, C. W., Mundlos, S. \& Bateman, J. F. A COL2A1 mutation in achondrogenesis type II results in the replacement of type II collagen by type I and III collagens in cartilage. J. Biol. Chem. 270, 1747-1753 (1995).

35. Strutz, F. et al. Identification and characterization of a fibroblast marker: FSP1. J. cell Biol. 130, 393-405 (1995).

36. Corada, M. et al. Monoclonal antibodies directed to different regions of vascular endothelial cadherin extracellular domain affect adhesion and clustering of the protein and modulate endothelial permeability. Blood 97, 1679-1684 (2001).

37. Armulik, A., Genove, G. \& Betsholtz, C. Pericytes: developmental, physiological, and pathological perspectives, problems, and promises. Developmental cell 21, 193-215 (2011).

38. Lim, J., Grafe, I., Alexander, S. \& Lee, B. Genetic causes and mechanisms of Osteogenesis Imperfecta. Bone 102, 40-49 (2017).

39. Kamoun-Goldrat, A. S., Le \& Merrer, M. F. Animal models of osteogenesis imperfecta and related syndromes. J. Bone Min. Metab. 25, 211-218 (2007)

40. Colnot, C., Thompson, Z., Miclau, T., Werb, Z. \& Helms, J. A. Altered fracture repair in the absence of MMP9. Development 130, 4123-4133 (2003).

41. Wang, X. et al. MMP9 regulates the cellular response to inflammation after skeletal injury. Bone 52, 111-119 (2013).

42. Baht, G. S., Vi, L. \& Alman, B. A. The role of the immune cells in fracture healing. Curr. Osteoporos. Rep. 16, 138-145 (2018).

43. Einhorn, T. A. \& Gerstenfeld, L. C. Fracture healing: mechanisms and interventions. Nat. Rev. Rheumatol. 11, 45-54 (2015).

44. Kovtun, A., Messerer, D. A. C., Scharffetter-Kochanek, K., Huber-Lang, M. \& Ignatius, A. Neutrophils in tissue trauma of the skin, bone, and lung: two sides of the same coin. J. Immunol. Res. 2018, 8173983 (2018).

45. Tsukasaki, M. \& Takayanagi, H. Osteoimmunology: evolving concepts in bone-immune interactions in health and disease. Nat. Rev. Immunol. 19, 626-642 (2019).

46. Kovtun, A. et al. The crucial role of neutrophil granulocytes in bone fracture healing. Eur. Cell Mater. 32, 152-162 (2016).

47. Kalajzic, Z. et al. Use of an alpha-smooth muscle actin GFP reporter to identify an osteoprogenitor population. Bone 43, 501-510 (2008).

48. Grcevic, D. et al. In vivo fate mapping identifies mesenchymal progenitor cells. Stem Cells 30, 187-196 (2012).

49. Matthews, B. G. et al. Analysis of alphaSMA-labeled progenitor cell commitment identifies notch signaling as an important pathway in fracture healing. J. Bone Min. Res. 29, 1283-1294 (2014).

50. Matthews, B. G. et al. Osteogenic potential of alpha smooth muscle actin expressing muscle resident progenitor cells. Bone 84, 69-77 (2016).
51. Niedermeyer, J. et al. Targeted disruption of mouse fibroblast activation protein. Mol. Cell Biol. 20, 1089-1094 (2000).

52. Niedermeyer, J. et al. Expression of the fibroblast activation protein during mouse embryo development. Int J. Dev. Biol. 45, 445-447 (2001).

53. Sillence, D. O., Barlow, K. K., Garber, A. P., Hall, J. G. \& Rimoin, D. L. Osteogenesis imperfecta type II delineation of the phenotype with reference to genetic heterogeneity. Am. J. Med Genet 17, 407-423 (1984).

54. Sillence, D. O., Senn, A. \& Danks, D. M. Genetic heterogeneity in osteogenesis imperfecta. J. Med Genet 16, 101-116 (1979).

55. Van Dijk, F. S., Pals, G., Van Rijn, R. R., Nikkels, P. G. \& Cobben, J. M. Classification of Osteogenesis Imperfecta revisited. Eur. J. Med Genet 53, 1-5 (2010).

56. Inubushi T., Nozawa S., Matsumoto K., Irie F., Yamaguchi Y. Aberrant perichondrial BMP signaling mediates multiple osteochondroma genesis in mice. JCI insight 2, e90049 (2017).

57. Bowen, M. E., Ayturk, U. M., Kurek, K. C., Yang, W. \& Warman, M. L. SHP2 regulates chondrocyte terminal differentiation, growth plate architecture and skeletal cell fates. PLoS Genet 10, e1004364 (2014).

58. Camacho, N. P. et al. The material basis for reduced mechanical properties in oim mice bones. J. Bone Min. Res. 14, 264-272 (1999).

59. Phillips, C. L. et al. Oim mice exhibit altered femur and incisor mineral composition and decreased bone mineral density. Bone 27, 219-226 (2000).

60. Cardinal, M. et al. Sclerostin antibody reduces long bone fractures in the oim/ oim model of osteogenesis imperfecta. Bone 124, 137-147 (2019).

61. Claes, L., Recknagel, S. \& Ignatius, A. Fracture healing under healthy and inflammatory conditions. Nat. Rev. Rheumatol. 8, 133-143 (2012).

62. Jikko, A., Harris, S. E., Chen, D., Mendrick, D. L. \& Damsky, C. H. Collagen integrin receptors regulate early osteoblast differentiation induced by BMP-2. J. Bone Min. Res. 14, 1075-1083 (1999).

63. $\mathrm{Li}, \mathrm{H}$. et al. Immature osteoblast lineage cells increase osteoclastogenesis in osteogenesis imperfecta murine. Am. J. Pathol. 176, 2405-2413 (2010).

64. Lynch, M. P., Stein, J. L., Stein, G. S. \& Lian, J. B. The influence of type I collagen on the development and maintenance of the osteoblast phenotype in primary and passaged rat calvarial osteoblasts: modification of expression of genes supporting cell growth, adhesion, and extracellular matrix mineralization. Exp. cell Res. 216, 35-45 (1995).

65. Xue, C., Plieth, D., Venkov, C., Xu, C. \& Neilson, E. G. The gatekeeper effect of epithelial-mesenchymal transition regulates the frequency of breast cancer metastasis. Cancer Res. 63, 3386-3394 (2003).

66. Bhowmick, N. A. et al. TGF-beta signaling in fibroblasts modulates the oncogenic potential of adjacent epithelia. Science 303, 848-851 (2004).

67. LeBleu, V. S. et al. Origin and function of myofibroblasts in kidney fibrosis. Nat. Med. 19, 1047-1053 (2013).

68. Chen, Y. et al. Type I collagen deletion in alphaSMA(+) myofibroblasts augments immune suppression and accelerates progression of pancreatic cancer. Cancer cell 39, 548-565 e546 (2021).

69. Chen Y., et al. Dual reporter genetic mouse models of pancreatic cancer identify an epithelial-to-mesenchymal transition-independent metastasis program. EMBO molecular medicine 10, e9085 (2018).

70. Zheng, X. et al. Epithelial-to-mesenchymal transition is dispensable for metastasis but induces chemoresistance in pancreatic cancer. Nature 527, 525-530 (2015).

\section{Acknowledgements}

We thank B. Lee at Baylor College of Medicine for helpful discussions, P.E. Phillips for the assistance with tissue sample processing and staining, V. Van, C.V. Kingsley, and K.L. Maldonado for the micro-CT and radiography at the Small Animal Imaging Facility of MDACC (Supported by the NIH/NCI under award number P30CA016672), K.M. Ramirez and R. Jewell at the South Campus Flow Cytometry Core Laboratory of MDACC for flow cytometry cell sorting (in part supported by NCI P30CA16672), E.J. Thompson, D.P. Pollock and Y. Chen at the Advanced Technology Genomics Core for their assistance for the single-cell RNA-sequencing analysis, in part supported by NCI CA016672 (ATGC), the Genetically Engineered Mouse Facility team of MDACC for the generation of Colla $1^{\text {loxP/loxP }}$ mouse strain. This is study was supported by MD Anderson Cancer Center and partly funded by the NCI grant P01CA117969.

\section{Author contributions}

Conceptualization: Y.C., R.K. Data curation: Y.C. Investigation: Y.C., S.Y., S.L., H.S. Methodology: Y.C., S.Y., C.G.A. Single-cell RNA-Seq Analyses: S.Y., Y.C. Resources: R.K. Supervision: Y.C., R.K. Validation: Y.C. Writing - original draft: Y.C. and R.K. Writing review \& editing: Y.C., S.Y., S.L., K.M.McA., H.S., R.K.

\section{Competing interests}

The authors declare no competing interests. 


\section{Additional information}

Supplementary information The online version contains supplementary material available at https://doi.org/10.1038/s41467-021-27563-3.

Correspondence and requests for materials should be addressed to Raghu Kalluri.

Peer review information Nature Communications thanks Guillaume Mabilleau and the other, anonymous, reviewer(s) for their contribution to the peer review of this work.

Reprints and permission information is available at http://www.nature.com/reprints

Publisher's note Springer Nature remains neutral with regard to jurisdictional claims in published maps and institutional affiliations. (c) (P) Open Access This article is licensed under a Creative Commons Attribution 4.0 International License, which permits use, sharing, adaptation, distribution and reproduction in any medium or format, as long as you give appropriate credit to the original author(s) and the source, provide a link to the Creative Commons license, and indicate if changes were made. The images or other third party material in this article are included in the article's Creative Commons license, unless indicated otherwise in a credit line to the material. If material is not included in the article's Creative Commons license and your intended use is not permitted by statutory regulation or exceeds the permitted use, you will need to obtain permission directly from the copyright holder. To view a copy of this license, visit http://creativecommons.org/ licenses/by/4.0/.

(C) The Author(s) 2021 